\title{
Large Cardinals, Inner Models, and Determinacy: An Introductory Overview
}

\author{
P. D. Welch
}

\begin{abstract}
The interaction between large cardinals, determinacy of two-person perfect information games, and inner model theory has been a singularly powerful driving force in modern set theory during the last three decades. For the outsider the intellectual excitement is often tempered by the somewhat daunting technicalities, and the seeming length of study needed to understand the flow of ideas. The purpose of this article is to try and give a short, albeit rather rough, guide to the broad lines of development.
\end{abstract}

\section{Introduction}

The following article grew out of a series of three tutorials at the conference on the development of large cardinal hypothesis and their connections to proofs of the determinacy of two-person perfect information Gale-Stewart games. The intention was to give a perspective, not to specialists in set theory, who in any case either would have no need for such or would know where to go find it, but to those interested in the foundations of set theory already with some technical knowledge of ZermeloFraenkel axiomatics and the construction of Gödel's universe $L$, but who may not have seen a systematic production of the hierarchy of increasing strong axioms of infinity arising from embeddings of the universe $V$ and in particular the connections of these axioms with the descriptive set theory of the real continuum. It was hoped that the lectures would provide a fast ascent without oxygen to some of the peaks of the last twenty years. In this we believed that a lot could be learned, not in detail, nor even attempting to provide a working knowledge (for which a longer-term devotion would be required, and again for which there are thorough texts) but that would bring about at least some familiarity with the technical tools, as well as some insight as to why they had been developed and how they had been used.

Received April 27, 2012; accepted June 8, 2014

2010 Mathematics Subject Classification: Primary 03A05; Secondary 03E55, 03E60, 03E45

Keywords: large cardinals, inner models, determinacy

(C) 2015 by University of Notre Dame 10.1215/00294527-2835083 
We should also like to urge the reader to study Jensen's masterly, and nontechnical, overview [11] if he or she has not already done so.

1.1 Overview The roots of modern set theory lie surprisingly deep in the history of the subject, although it is admittedly rather young compared to other areas of mathematics. It is well known that it concerns itself still with the question that vexed its founding father: Cantor's continuum problem (or hypothesis, hence $\mathrm{CH}$ ), which states that any set of real numbers is either equinumerous with all of $\mathbb{R}$ or is countable. However some of the early analysts such as the those from the French school, Lebesgue, Borel, Baire, and then the Russians, Luzin and more particularly Suslin, introduced both further questions and new methods of representation that are still key today. Today descriptive set theory is that branch of set theory which seeks to investigate, not the whole real continuum but definable parts or fragments of it. In doing this two things are achieved: (i) one may concentrate on perhaps simpler parts of the continuum for which the questions are more tractable, and (ii) the definable parts are rather concretely given, and as such the results proven may give more definite information to the analyst who is uninterested in foundational questions. At the same time this gives clues to the most general question concerning the whole continuum. The roots of descriptive set theory can also be traced back to Cantor himself: he first tried (and succeeded) to solve the $\mathrm{CH}$ for closed sets. His ambition was to proceed through increasingly complicated sets until, somehow, he had conquered the whole continuum. He, and Bernstein, showed that a closed set is the union of a perfect set with a countable set of isolated points. Thus closed sets enjoyed the "perfect subset property" (PSP). CH for closed sets follows: if a closed set is uncountable the perfect part is nonempty and can easily be shown to be equinumerous with $\mathbb{R}$. Borel defined a hierarchy of more complicated sets: one starts with the class of open sets at the base level and then builds up levels by the process of taking complementation and countable unions of the sets created so far. This Borel hierarchy is built in precisely $\omega_{1}$-many stages, and was much studied. It was found that PSP holds for Borel sets.

Suslin then showed the same for the analytic, or $\boldsymbol{\Sigma}_{\mathbf{1}}^{\mathbf{1}}$-sets. More detailed definitions are below, but an analytic subset of $\mathbb{R}$ (depicted on the $x$-axis) is one that is the projection of a Borel set in the $(x, y)$-plane down to that $x$-axis. This can be seen as a further step in Cantor's original plan: now any analytic set satisfies $\mathrm{CH}$. However the program stopped there. Later Gödel was to show that in $L$, his constructible hierarchy, although $\mathrm{CH}$ was true, coanalytic or $\boldsymbol{\Pi}_{\mathbf{1}}^{\mathbf{1}}$-sets failed to have the PSP. Hence it was consistent with the axioms of ZFC that PSP failed at this level. Hence the analysts had gone as far as they could have done using analytical techniques. However, the PSP is only an example of one of several properties. The property of being Lebesgue measurable (LM) was also shown to hold for first Borel, then analytic (and since complements of measurable sets are measurable too, also coanalytic) sets. The case is similar for the Baire property (BP, i.e., a set $A$ with the Baire property is almost an open set, in that there is an open set $O$ so that the symmetric difference of the two sets $A$ and $O$ is "thin" in the sense of being the union of countably many nowhere dense sets), and moreover for the uniformization property: that coanalytic regions of the plane could be uniformized by coanalytic functions. Together this clutch of properties became (more recently) collectively known as the regularity properties. Again the BP and LM could both consistently fail for the same reason as for the PSP: in the Gödel universe $\boldsymbol{\Delta}_{\mathbf{2}} \mathbf{1}$-sets failed to have them. 
Proceeding in another direction Banach had considered Lebesgue's problem of defining a translation-invariant countably additive measure on $\mathbb{R}$. He replaced the translation-invariance part of the requirement with that of asking the measure of a single point to be nonzero. Was such a measure possible? Again this was ruled out by an axiom of choice construction, as Vitali had shown would happen for any measure satisfying Lebesgue's requirements. However Banach's requirements allowed one to generalize away from $\mathcal{P}(\mathbb{R})$ to consider measures on $\mathcal{P}(S)$ for any set $S$ (Banach [1]). The additivity of such a measure, were it to exist, would be that of the cardinality of $S$. If such existed for an uncountable set $S$ with $|S|=\kappa$, then $\kappa$ was called real-valued measurable. Ulam [36] showed that a real-valued measurable cardinal was weakly inaccessible in Hausdorff's sense. If the measure was atomless, then $2^{\aleph_{0}}>\kappa$. In this way large cardinals and measures enter the scene. Ulam also defined the ultimately more consequential notion of a 2-valued measure $\kappa$-additive measure. With Tarski the cardinals $\kappa$ admitting such measures on their power sets were shown to be strongly inaccessible, but not until the work of Hanf [9] was it shown that the least measurable cardinal could not be the least inaccessible.

Shortly thereafter Scott [31] used the technique of ultrapowers from model theory to show that the existence of a measurable cardinal implied $V \neq L$ (see Theorem 2.5 below). This technique has become ubiquitous in modern set theory, with the realization that a measurable cardinal allows an ultrapower of the whole universe $V$ into some proper inner model $M$, with a first-order truth-preserving elementary embedding: $j: V \longrightarrow_{e} M$. Embeddings of the universe $V$ with stronger properties arose from the work of Solovay and of Reinhardt, and these were defined using ultrafilters on different base sets. The cardinals that were the first point moved, or the critical point of such embeddings was called supercompact, and the embeddings they produced had markedly different aspects to those from simple measures alone. Together with large cardinals defined from other differing directions, these would eventually all be seen to lie in a well-ordered spectrum of increasing strength giving rise to stronger and stronger embedding properties, which to a first approximation can be said to be about how much of $V$ is carried over into the target model $M$. (We give definitions of the more prominent of these again below.)

"Inner models" in this account are transitive class models of ZFC. (This can be given a first-order formulation.) Gödel's $L$ was the first inner model to be discovered (besides $V$ itself, of course). Relativized constructibility is easy to define: one enlarges the language of set theory to include (one or more) additional predicates into the first-order definability operator. Thus models $\langle L[\mu], \in, \mu\rangle$ where $\mu$ is a twovalued $\kappa$-additive measure on some $\kappa$ may be defined. Solovay defined such, and their properties were investigated by Silver and Kunen. $L[\mu]$ was seen to be a generalization of $L$ : the generalized continuum hypothesis $(\mathrm{GCH})$ held, and there was a global well order of its universe. The definability of that well order on its real continuum was, however, $\Delta_{3}^{1}$ rather than $\Delta_{2}^{1}$ which had been the case for $L$.

Other generalized notions of definability were possible: instead of definability relative to a predicate one may start with a set $R$ and define

$$
\begin{gathered}
L_{0}(R)=R, \quad L_{\alpha+1}(R)=\operatorname{Def}\left(\left\langle L_{\alpha}(R), \in\right\rangle\right), \\
\operatorname{Lim}(\lambda) \longrightarrow L_{\lambda}(R)=\bigcup_{\alpha<\lambda} L_{\alpha}(R) .
\end{gathered}
$$


The universe $L(R)=\bigcup_{\alpha \in O n} L_{\alpha}(R)$ is then an inner model of ZF (not necessarily $\mathrm{AC}$ as there is not necessarily a well order of $R$ definable over any of the levels $\left.L_{\alpha}(R)\right)$. For $R=\mathbb{R}$ this has been a model of tremendous importance as we shall see. One can regard it as a laboratory for very generalized notions of definability over analysis: it contains the projective sets of analysis right down at the bottom and allows for definitions over analysis but containing more and more ordinals.

There was a further striking result concerning measurability.

Theorem 1.1 (Solovay [33]) (ZFC) If there is a measurable cardinal, then all $\mathbf{\Sigma}_{\mathbf{2}} \mathbf{1}$-sets of reals have the regularity properties of LM, BP, and PSP.

This was probably the first indication that large cardinals could really influence how the continuum would look to an analyst: by assuming some measure on some remote cardinal the nature of $V_{\omega+1}$ is fundamentally different.

However researchers were already deriving the regularity properties from a quite unexpected quarter. This was the notion of a two-person, perfect-information game (see Definition 3.1 below). Curiously, Zermelo had first published on the existence of winning strategies for finite games, but Polish mathematicians such as Steinhaus, Banach, and Mazur in the 1920s took up the question of infinite length games (see the historical remarks in [6, Section $6 \mathrm{H}]$ ). As an example such a game $G_{A}$ is based on a subset $A \subseteq \omega^{\omega}$ (the "payoff set") and then two players alternate choosing integers $n_{0}, m_{0}, n_{1}, m_{1}, \ldots$ (with perfect knowledge of the other's moves) and after $\omega$-many moves, the mutually constructed infinite sequence $z \in \omega^{\omega}$ may or may not lie in $A$. If it does, player $I$ is deemed to have won. Otherwise player $I I$ wins.

It was then asked for which payoff sets $A$ could a player have a winning strategy (in the obvious sense)? In their formulations $\mathbb{R}$ was taken as $\omega^{\omega}$ with moves taken from $\omega$. In descriptive set theory it is usual to consider the reals as $\omega^{\omega}$ rather than the Euclidean $\mathbb{R}: \omega^{\omega}$ is homeomorphic to the irrationals, and only the countable set of rationals is "omitted." Moreover the topology determined by finite sequences (see below) is very conducive to descriptive set-theoretical arguments: the resulting Baire space is an example of a well-studied space, being a Polish space, that is, a separable complete metric space; moreover it is homeomorphic to the direct product of any finite number of copies of itself. Questions of dimension are thus made irrelevant: proving a result about the descriptive set theory of the one-dimensional space $\omega^{\omega}$, in general one has the result for the $k$-dimensional $\left(\omega^{\omega}\right)^{k}$.

With this topology one sees that if $A \subseteq \omega^{\omega}$ is an open set, and player $I$ has a winning strategy, then any run of the game using that strategy has essentially been won by $I$ by a finite stage. Mazur had already conjectured the connection between the class of $A$ for which the games $G_{A}$ are determined games (meaning one of the players has a winning strategy) and the Baire property for that class. This was proven by Banach in 1935 .

We now know that determinacy implies the regularity properties. Let $\Gamma$ be a class of subsets of $\omega^{\omega}$. If $G_{A}$ is determined for all $A \in \Gamma$ we write $\operatorname{Det}(\Gamma)$. If $\Gamma$ has some minimal closure properties (this is "adequacy" in Moschovakis's terminology; see [26]) which we shall not define here, but are enjoyed by all the definable projective classes $\Sigma_{n}^{1}, \Pi_{n}^{1}$, and so forth, then as the combined work of Banach, Mazur, Oxtoby (BP), Mycielski-Swierczkovski [27] (LM), Davis [2] (PSP) (all by 1964) showed, we have the following. 
Theorem $1.2(\mathrm{ZFC}) \quad$ Assume $\operatorname{Det}(\Gamma)$. Then all sets in $\Gamma$ have the regularity properties BP, LM, and PSP.

Actually some more can be extracted from these proofs: assuming $\operatorname{Det}(\Gamma)$ the point class $\exists^{\mathbb{R}} \Gamma$ also enjoys these regularity properties. Here $\exists^{\mathbb{R}} \Gamma$ is the point class of projections of sets $A \in \Gamma$. (Here it is particularly useful to be able to prove results simultaneously in any finite dimension.) That is, $B \in \exists^{\mathbb{R}} \Gamma$ if and only if $\exists A \in \Gamma B=\{x \mid \exists y(x, y) \in A\}$.

By Martin's result on Borel determinacy, in other words, that ZFC $\vdash \operatorname{Det}\left(\boldsymbol{\Delta}_{\mathbf{1}}^{\mathbf{1}}\right)$ (see Theorem 3.8 below), we thus have the regularity properties for $\exists^{\mathbb{R}} \boldsymbol{\Delta}_{\mathbf{1}}^{\mathbf{1}}=\mathbf{\Sigma}_{\mathbf{1}}^{\mathbf{1}}$ (see below for the definition of these classes within the projective hierarchy; in brief the projective sets are those definable in analysis, that is, definable over the standard model of second-order number theory). The conclusion of the regularity properties for $\boldsymbol{\Sigma}_{\mathbf{1}}^{\mathbf{1}}$ is of course nothing more than the work of the earlier analysts. However, now we have that $\operatorname{Det}\left(\boldsymbol{\Pi}_{\mathbf{1}}^{\mathbf{1}}\right)$ yields them for $\boldsymbol{\Sigma}_{\mathbf{2}}^{\mathbf{1}}$.

In short, determinacy is, unexpectedly perhaps, yielding the regularity properties on Baire space (and this transfers to other complete Polish spaces, such as Cantor space $2^{\omega}$, as well as back to the Euclidean $\mathbb{R}$ ).

Solovay had obtained in 1965 (as mentioned above) the very same $\mathbf{\Sigma}_{\mathbf{2}}^{\mathbf{1}}$-regularity results from a measurable cardinal. It turned out that determinacy for coanalytic sets follows too.

\section{Theorem 1.3 (Martin [19]) (ZFC) The existence of a measurable cardinal implies $\operatorname{Det}\left(\boldsymbol{\Pi}_{\mathbf{1}}^{\mathbf{1}}\right)$.}

It turned out that $\operatorname{Det}\left(\Pi_{1}^{1}\right)$ was equivalent to a nontrivial elementary embedding of $L$ to $L$ (the Martin-Harrington theorem; see Theorem 3.9 below).

Martin [21] later went on to show that $\operatorname{Det}\left(\boldsymbol{\Pi}_{\mathbf{2}}^{\mathbf{1}}\right)$ followed from the existence of a nontrivial elementary embedding $j: V_{\lambda} \longrightarrow_{e} V_{\lambda}$. This is one of the strongest hypotheses known for a large cardinal property, and it led to the feeling that PD, the determinacy of all point classes in the projective hierarchy was stronger than all large cardinal hypotheses then known. This was amplified by Woodin's result that PD was indeed provable from a hypothesis even stronger than the one Martin used. Later results, coming from quite a different direction, of Foreman, Magidor and Shelah showed that a supercompact cardinal (defined below) implied that all sets of reals in $L(\mathbb{R})$ were Lebesgue measurable. This hypothesis was much weaker than the Martin-Woodin hypotheses and opened up the possibility that perhaps PD might not be so strong after all, since $L M$, being one of its consequences, was not.

So, it was after these results that it was discovered that embeddings of proper inner models (not of the whole of $V$ into an inner model) are equivalent, broadly speaking, to that of the determinacy of point classes within the projective hierarchy (although such had been established for some point classes strictly within $\boldsymbol{\Delta}_{\mathbf{2}}^{\mathbf{1}}$ ). In the case of a $\Pi_{1}^{1}$-set $A$ the point was to use a representation of $A$ as a tree on (finite sequences from) $\omega \times \omega^{<\omega}$ in an auxiliary open game. The latter game would be in a different space of moves but importantly would be an open game there and thus would (in ZFC) have a winning strategy $\sigma^{*}$. Indiscernibility (from Silver's indiscernibles from the embedding of $L$ to $L$ ) was used to "integrate out" a strategy $\sigma$ for the original integer game $G_{A}$ from $\sigma^{*}$. This rough scheme applies for the higher point classes $\Pi_{n}^{1}$ : homogeneous tree representations of the set $A$ were now on $\omega \times \omega^{<\lambda}$ for 
larger ordinals $\lambda$ and the homogeneity guarantees that there are sufficiently coherent towers of measures on the tree to run the argument. A subtle kind of cardinal was defined, the Woodin cardinal, which allowed the correct properties of these trees to be propagated through the increasing complexity of the projective hierarchy.

When the dust settled, we had the following.

Theorem 1.4 (Martin and Steel [23]) (ZFC) The existence of $n$ Woodin cardinals with a measurable above them all, implies $\operatorname{Det}\left(\boldsymbol{\Pi}_{n+\mathbf{1}}^{\mathbf{1}}\right)$.

Theorem 1.5 (Martin and Steel [23], Woodin 1985) (ZFC) The existence of infinitely many Woodin cardinals with a measurable cardinal above them all, implies $\mathrm{AD}^{L(\mathbb{R})}$.

The notion of a Woodin cardinal is much weaker than a supercompact, so the original thought concerning the consistency strength of PD turned out to be quite incorrect. In fact, an exact equiconsistency obtains (see Theorem 3.19 below). Now we have that large cardinals are sufficient to prove definable determinacy, and that conversely definable determinacy implies the existence of inner models of those large cardinals. The question arises as to whether we should wish to adopt definable determinacy as a new "axiom." For a full account of this discussion the reader is referred to the excellent account of Koellner's [14], but we summarize as follows.

There is now a point of view that there is a case for assuming projective determinacy, or $\mathrm{PD}$, as a natural "completion" for the theory of the hereditarily countable sets: if one takes the theory $\mathrm{ZFC}^{-}+\mathrm{PD}+$ "Every set is countable," then this theory proves that all projective sets of reals have the regularity properties. Moreover the only sentences (as yet) known to be independent of the theory are Gödel-like sentences (see Woodin [38], [39]). If we move to a more global perspective and consider $\mathrm{AD}^{L(\mathbb{R})}$, then we have a very robust phenomenon concerning it: it appears (empirically) that any theory extending ZFC of sufficient strength implies it outright (not just its consistency, we emphasize). This is the case of course of theories that are also themselves mutually inconsistent when put together, but they all have $\mathrm{AD}^{L(\mathbb{R})}$ as part of their common core consequences. If we were ever to choose one such theory as constituting a new "axiom" we should be sure to get $\mathrm{AD}^{L(\mathbb{R})}$. Woodin has also shown that $\mathrm{AD}^{L(\mathbb{R})}$ is remarkable in that it is also implied by its consequences.

Theorem 1.6 (Woodin) (ZFC) If all sets of reals enjoy the LM and PSP properties, and $\operatorname{Unif}\left(\Sigma_{1}^{2}\right)$ also holds, then $\mathrm{AD}^{L(\mathbb{R})}$.

The latter uniformization property also holds in $L(\mathbb{R})$ assuming $\mathrm{AD}$ there. We thus cannot find a different theory or axiom implying the regularity consequences in $L(\mathbb{R})$ without automatically getting AD there. Koellner in [14] makes a comparison with an empirical science: we are remarkably obtaining back from our "empirical consequences" of the theory, that is, the observations of the regularity properties, the theory itself. This of course does not happen in physical theories, but the very remarkability of this phenomenon is one reason why many set theorists, especially those on or near the realist wing, feel that determinacy hypotheses such as $\mathrm{AD}^{L(\mathbb{R})}$ are compelling assertions about the set-theoretical universe.

Woodin has also shown that large cardinals in inner models are obtainable from determinacy hypotheses.

Theorem 1.7 (Woodin) (ZFC) The theories $\mathrm{ZF}+\mathrm{AD}$ and $\mathrm{ZFC}+$ "There exist infinitely many Woodin cardinals" are equiconsistent. 
There are some equivalents over ZFC for levels of projective determinacy which we shall not detail here (cf. Koellner and Woodin [15]).

Woodin has also demonstrated a very impressive array of results concerning attempts to develop a logic, the $\Omega$-logic, that would factor out the baleful effects of the set-forcing independence results. One is thus interested in sentences, theories, and so forth which survive attempts to alter their truth value by forcing arguments. Such sentences are deemed "generically absolute."

Theorem 1.8 (Woodin) (ZFC) Assume that there is a proper class of Woodin cardinals. Then the (first-order) theory of $L(\mathbb{R})$ is generically absolute. It thus cannot be altered by any set forcing.

See Theorem 3.20 below. In particular as it only requires infinitely many Woodin cardinals to establish that $\mathrm{AD}^{L(\mathbb{R})}$, and as any set forcing will still leave infinitely many Woodin cardinals intact, every attempt to build a model using (set) forcing techniques will still result in a generic extension in which $\mathrm{AD}$ holds in the $L(\mathbb{R})$ of that generic extension. Now compare that with the following.

Theorem 1.9 (Woodin) (ZFC) Suppose that there is a proper class of inaccessible cardinals and that the theory of $L(\mathbb{R})$ is generically absolute. Then $\mathrm{AD}^{L(\mathbb{R})}$ holds.

As with Theorem 1.6, the last theorem can also be viewed as another recovery theorem: $\mathrm{AD}^{L(\mathbb{R})}$ is being recovered from its consequences. Here we are witnessing that it must hold if the kind of generic absoluteness that flows from the existence of large cardinals holds true.

1.2 Preliminaries Our notation and formalisms are quite standard and can be found in many textbooks, but in particular we mention Jech [10] and Kanamori [12]. We let $\mathscr{L}_{\dot{\epsilon}}$ denote the first-order language of set theory; by ZF we mean a formulation of first-order Zermelo-Fraenkel. We occasionally mention the theory $\mathrm{ZF}^{-}$, which is $\mathrm{ZF}$ with the power set axiom dropped. For $\mathrm{ZF}^{-}$it is usual to include the collection scheme, rather than the replacement scheme, as the latter without power set is too weak to prove some basic facts.

By $\mathscr{L}_{\dot{\epsilon}, \dot{\mathcal{A}}}$ we mean the standard language of set theory with an (optional) predicate $\dot{A}$. In both these languages we freely make use of abstract terms $t=\{z \mid \varphi(\vec{y}, z)\}$ as if they were part of the languages. $\mathrm{ZF}_{A}$ is then a formulation of $\mathrm{ZF}$ with instances of the predicate $\dot{A}$ allowed in the axioms. A set is transitive, $\operatorname{Trans}(x)$, if every element of $x$ is at the same time a subset of $x$.

The class of all ordinals is denoted On; Sing is the class of singular ordinals; Card is the class of all cardinals (we assume AC throughout and that cardinals are initial ordinals). SingCard, Reg are the classes of singular cardinals and regular cardinals, respectively. Inacc is the class of (strongly) inaccessible cardinals. For a limit ordinal $\tau$, by the "cofinality of $\tau$ " (cf. $(\tau)$ ) is meant the least $\delta$ such that there is a function $f: \delta \longrightarrow \tau$ with $\operatorname{ran}(f)$ unbounded in $\tau(\tau \in \operatorname{Reg}$ then, if $\operatorname{cf}(\tau)=\tau)$. The rank function $\rho(x)=\alpha$ is $\Delta_{1}^{\mathrm{ZF}}$, and the relation of $y$ and $\alpha, y=V_{\alpha}$, is $\Pi_{1}^{\mathrm{ZF}}$. We let ${ }^{x} y$ denote the set of all functions from the set $x$ to $y$. It is often customary to deviate from the practice when $x=\omega$ and we are considering, for example, ${ }^{\omega} \omega$ or ${ }^{\omega} X$ as spaces, and then we shall write $\omega^{\omega}$ or $X^{\omega}$.

It is useful to fix a recursive enumeration $\left\langle\tau_{i} \mid i<\omega\right\rangle$ of Seq $=_{\mathrm{df}}{ }^{<\omega} \omega$ so that
(i) $\tau_{0}=(), \tau_{1}=(0)$;
(ii) $i \geq\left|\tau_{i}\right|\left(=_{\mathrm{df}} \operatorname{lh}\left(\tau_{i}\right)\right)$;
(iii) $\tau_{i} \subset \tau_{j} \longrightarrow i<j$. 
(We may similarly define recursive enumerating sequences $\left\langle\tau_{i}^{k} \quad \mid i<\omega\right\rangle$ for $^{k}\left({ }^{<\omega} \omega\right)$.)

The Kleene-Brouwer ordering is defined as follows: for $s, t \in{ }^{<\omega}$ On put

$$
s<<_{\mathrm{KB}} t \leftrightarrow s \supset t \vee(s(i)<t(i) \quad \text { where } i \in \omega \text { is least with } s(i) \neq t(i)) .
$$

Finally, recall that a set $B \subseteq \mathbb{R}$ is $\Pi_{n}^{1}$ if it can be defined in $Z_{2}$ with

$$
x \in B \leftrightarrow \exists f_{1} \forall f_{2} \cdots Q f_{n} \forall k R\left(k, \overrightarrow{f_{i} \uparrow k}, \bar{x} \uparrow k\right) \quad \text { with } R \in \Sigma_{1}^{0}
$$

with the $f_{i} \in \mathbb{N}^{\mathbb{N}}$ and $k \in \mathbb{N}$. ( $Z_{2}$ is a formalization of second-order number theory, or analysis; see Simpson [32].) A set is $\Pi_{n}^{1}(a)$ for some $a \in \mathbb{R}$ if the parameter $a$ is allowed into the above defining $\Sigma_{1}^{0}$-relation $R$. The class $\boldsymbol{\Pi}_{\boldsymbol{n}}^{\mathbf{1}}$ is the union over $a$ of the classes $\Pi_{n}^{1}(a)$. As the typeface indicates, a boldface point class indicates that, in general, real parameters are present in the definition of a member of that class. A lightface point class such as $\Sigma_{1}^{1}$ (also called lightfaced analytic), or $\Pi_{n}^{1}$, is one which involves no such parameters. As is usual in set theory, we make little distinction between $\mathbb{R}$ and $\omega^{\omega}$, or even $\mathcal{P}(\omega)$ on occasion.

\section{Inner Models, Elementary Embeddings, and Covering Lemmas}

Gödel constructed an inner model of $V$ (here the hierarchy of well-founded sets $\left.\mathrm{WF}=\bigcup_{\alpha \in \mathrm{On}} V_{\alpha}\right)$ by taking a "definable power set" operation $x \longrightarrow \operatorname{Def}(x)$, where $x$ is shorthand for the first-order structure $\langle x, \in\rangle=\langle x, \in \mid x \times x\rangle$. By the parsimonious nature of the Def function Gödel showed that the sets so constructed were very regular, that they appeared in a definable order (thus a global axiom of choice was verified to hold in $L$ ), and that few subsets of any cardinal were admitted; the last justifies the $\mathrm{GCH}$, the generalized continuum hypothesis as holding in $L$.

The Def function can be augmented as a relativized definability operation $x \longrightarrow \operatorname{Def}_{A}(x)$ using definability over $\langle x, A \uparrow x, \in \uparrow x \times x\rangle$ in the wider

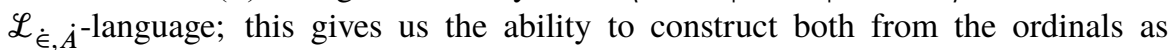
well as using information from the predicate $A$. These two definability functions are given by $\Delta_{1}^{\mathrm{ZF}^{-}}$- or $\Delta_{1}^{\mathrm{ZF}_{\mathrm{A}}^{-}}$-terms and hence are absolute. Given a model $W$ (thus given by some class term) we define the relativization of a formula $\varphi$ (or a theory $T$ ) denoted by $\varphi^{W}$ (resp., $T^{W}$ ), to be the formula $\varphi$ with all quantifiers $\forall v_{i}, \exists v_{j}$ replaced by $\forall v_{i} \in W, \exists v_{j} \in W$ (resp., the same for all formulae in $T$ ). We think of this as expressing that the formula $\varphi$ (or theory $T$ ) holds in $W$.

We let $V=L$ abbreviate the statement that every set is constructible, or more formally: $\forall x \exists \alpha\left(x \in L_{\alpha}\right)$. That $(V=L)^{L}$ was shown by Gödel [7], as well as $(\mathrm{AC})^{L},(\mathrm{GCH})^{L}$ thereby establishing: $\mathrm{Con}(\mathrm{ZF}) \Longrightarrow \mathrm{Con}(\mathrm{ZFC}+\mathrm{GCH})$. Why should $V=L$ ? As this is a hierarchy of sets constructed by syntactico-semantical means, and not responding to any particular intuitions about set existence, most set theorists do not believe $V=L$ (but see the discussion in [11]).

We shall see good reasons for asking whether there are other inner models, some associated with the notions of elementary embeddings. We first define our terms.

Definition 2.1 (Inner model of ZF) We have

$$
I M(M) \leftrightarrow \operatorname{Trans}(M) \wedge \text { On } \subseteq M \wedge(\mathrm{ZF})^{M} .
$$


In the above we are assuming that $M$ is given by some term of the language. The notion of being an inner model of ZF actually has a first-order formalization: it is well known (see [10]) that

$$
\mathrm{ZF} \vdash I M(M) \leftrightarrow \forall u \subseteq M \exists v \supseteq u(\operatorname{Trans}(v) \wedge \operatorname{Def}(\langle v, \in\rangle) \subseteq M) .
$$

The notion of an inner model of ZFC is simply the above with AC added at the relevant points.

We single out the following beautiful theorem as the motivation for this account.

Theorem $2.2(\mathbf{Z F}) \quad$ The following are equivalent:

(a) $\exists j: L \longrightarrow_{e} L$;

(b) $\exists \gamma\left(\omega_{2} \leq \gamma \in \operatorname{SingCard} \wedge(\gamma \notin \operatorname{SingCard})^{L}\right)$;

(c) $\operatorname{Det}\left(\Pi_{1}^{1}\right)$.

Here (a) expresses that there is a truth-preserving map between $L$ and itself (see Definition 2.3 below) which is nontrivial: that is, it is not the identity map. This is actually a culmulative theorem established over several years: (a) $\leftrightarrow$ (b) (Devlin and Jensen [4]); (a) $\rightarrow$ (c) (Martin [19]); (c) $\rightarrow$ (a) (Harrington [8]). Part (a) of the above theorem is sometimes stated as $0^{\#}$ exists. For the $(\mathrm{c}) \rightarrow($ a) direction, Martin actually showed that the determinacy of games at the third level of the difference hierarchy of analytic sets proved the existence of $0^{\#}$. This was then reduced by Harrington to a single analytic in the work cited. For an elegant proof of the Harrington result see Sami [28].

One should consider the overall form of this theorem: even if it is anachronistically stated, it is a deep result; it takes concepts that a priori have no connection with each other but by deep analysis are shown to be equivalent. The first, (a), involves an elementary embedding of $L$ to itself.

Much large cardinal theory is about which ultrafilters can or do exist on (large) sets; in particular, when those large sets are the power set of some cardinal of an inner model, then there is usually an equivalent formulation in terms of elementary embeddings, as defined below, of that inner model such as stated at (a).

It is often stated that $0^{\#}$ exists is the first large cardinal axiom, in that it transcends Gödel's $L$ in a drastic fashion: such embeddings cannot be added by Cohen-style forcing arguments and so can never be established by using small large cardinals where the latter are those consistent with the universe being $L$ itself (such as inaccessible, weakly compact, or ineffable cardinals, etc.). Indeed from properties of $0^{\#}$, one can show that the $L$ becomes a very thin inner model of $V$, and in some way becomes very similar to the class of ordinals alone.

The second clause, (b), is the negation of a form of the so-called covering lemma due to Jensen. What is being negated is the assertion that all singular cardinals are seen by $L$ to be singular: whilst $L$ may have many "cardinals" that it does not see, or have, functions for mapping them to smaller ordinals, functions that may exist in $V$, the assertion is that, nevertheless, $L$ can recognize the singularity of any singular cardinal of $V$. It must then possess functions cofinal in these cardinals. If this failed for just one singular cardinal, then $0^{\#}$ would exist. The covering lemma (which has stronger forms with more ramifications-see below) then asserts that the cardinality and cofinality structure of $V$ is reflected inwardly to some extent into that of $L$. If (a) holds however, this picture entirely collapses. The final clause (c) is then at first glance quite extraordinary: we are asked to believe that the determinacy of coanalytic 
games, thus something right down at the bottom of the ramified hierarchy of sets, has a global effect: that it engenders an elementary embedding $j: L \longrightarrow_{e} L$. It seems to have nothing to do with the other two clauses.

It is one of the triumphs of the California school of set theory in the 1980s that this picture relating large cardinals and determinacy for the projective point classes, and on to sets in $L(\mathbb{R})$ came fully into focus, and was conclusively analyzed. The equivalences in the theorem thus also show that determinacy is not some arcane curiosity in the set theorists' Wunderkammer but holds a central role in our current understanding of what the subject is about.

We explore the background to Jensen's (a) $\leftrightarrow$ (b). Again we have to define some terms.

Definition 2.3 (i) Let $M, N$ be inner models of ZF; $j: M \longrightarrow N$ is an elementary embedding if the function $j$ takes elements $x \in M$ to elements $j(x) \in N$ in a "truth-preserving way": for any formula $\varphi\left(v_{0}, \ldots, v_{n-1}\right)$ and any $\vec{x}=x_{0}, \ldots, x_{n-1} \in M$, then

$$
\varphi(\vec{x})^{M} \leftrightarrow \varphi(\overrightarrow{j(x)})^{N} .
$$

In this case we write: $j: M \longrightarrow_{e} N$. (We shall always assume that $j \neq \mathrm{id}$, and shall write $\operatorname{cp}(j)$ for the critical point: the least ordinal $\alpha$ such that $j(\alpha)>\alpha$, if it exists.)

(ii) If the above holds, but with the formulae restricted to a certain class, for example, the $\Sigma_{k}$ formulae, then we write $j: M \longrightarrow \Sigma_{k} N$.

In the above scheme, we have assumed that the models $M, N$ satisfy $I M(M)$, $I M(N)$ above and are given by terms of our basic set-theoretical language, and the same holds true for $j$. Our embeddings in this paper will all have critical points in the ordinals. It is an easy consequence of the $\mathrm{ZF}$-axioms (using the definition of the rank function, the $V_{\alpha}$-hierarchy, and replacement) that if $j: M \longrightarrow \Sigma_{1} N$, then by a (metatheoretic) induction on $k$ we may prove $j: M \longrightarrow \Sigma_{k} N$ for any $k \in \omega$.

If $\exists j: L \longrightarrow_{e} L$, then we may define a derived measure $U=U_{j}$ on $\kappa=\operatorname{cp}(j)$ as follows: we set

$$
X \in U_{j} \leftrightarrow X \in \mathcal{P}(\kappa)^{L} \wedge \kappa \in j(X) .
$$

Then $U_{j}$ is a normal measure (see [12, p. 52]) on $\mathcal{P}(\kappa)^{L}$. Suppose that we have $j: M \longrightarrow_{e} N, \operatorname{cp}(j)=\kappa$, and define $U=U_{j}$. Then we construct an ultrapower defining

$$
|\operatorname{Ult}(M, U)|=\left\{[f]_{\sim}: f \in{ }^{\kappa} M \cap M\right\},
$$

where

$$
f \sim g \leftrightarrow\{\alpha \mid f(\alpha)=g(\alpha)\} \in U
$$

and on which we can define a pseudo- $\epsilon$ relation $E$ :

$$
f E g \leftrightarrow\{\alpha \mid f(\alpha) \in g(\alpha)\} \in U .
$$

Because of where $U_{j}$ comes from, we are guaranteed that $E$ is well founded on $\operatorname{Ult}(M, U)$; we may define by recursion along $E$ the Mostowski-Shepherdson tran- 
sitivizing collapse isomorphism $\pi:\left(\operatorname{Ult}\left(M, U_{j}\right), E\right) \longrightarrow(\bar{N}, \in)$ with $\bar{N}$ transitive, and we have, with $k(\pi([f]))=j(f)(\kappa)$,

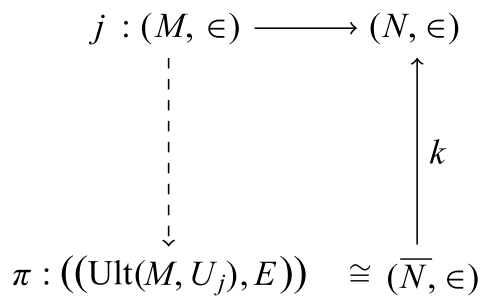

We can, and often do, have $(M, \in)=(V, \in)$. Also, starting from any $\kappa$-complete $U$ on $\mathcal{P}(\kappa)^{V}$ (for an uncountable regular $\kappa$ ), then we can define $\operatorname{Ult}(V, U)$ and we may prove outright that the $E$-relation on $\operatorname{Ult}(V, U)$ will be well founded. In general for well-founded cases we may then define the map $\pi$ as above.

Taking these facts together then, we have the following.

Theorem 2.4 (ZFC) Let $\kappa>\omega$. The following are equivalent:

(a) there is a $\kappa$-complete nonprinciple ultrafilter on $\mathcal{P}(\kappa)$;

(b) $\exists j: V \longrightarrow_{e} M$ with $\mathrm{cp}(j)=\kappa$.

Scott took the construction of an ultrapower from model theory and used it to prove the first theorem demonstrating that a "large cardinal" was incompatible with $V=L$.

Theorem 2.5 (Scott [31]) (ZF) We have

$$
\exists \kappa(\kappa \text { a measurable cardinal }) \Longrightarrow V \neq L .
$$

Proof If $V=L$, let $\kappa$ be the least such measurable cardinal (MC), form the ultrapower and so the embedding above. Then from $j: V \longrightarrow_{e} N$ and elementarity we have $(V=L)^{V} \longrightarrow(V=L)^{N}$; so as $\operatorname{Trans}(N), N=L$. However, " $\kappa$ is the least MC" implies that (" $j(\kappa)$ is the least MC") ${ }^{N}$. However, $N=V \wedge j(\kappa)>\kappa$ !

The assumption of this theorem implies $\exists j: V \rightarrow_{e} M$, but again by Gödel's results on the absoluteness of the $L$-construction, $L^{M}=L$, so $j \uparrow L: L \longrightarrow_{e} L$. Note that no first-order formula $\varphi\left(v_{0}\right)$ can differentiate between $\kappa$ and $j(\kappa)$ : $\varphi(\kappa)^{L} \leftrightarrow \varphi(j(\kappa))^{L}$. Moreover, both ordinals are inaccessible cardinals in the sense of $L$.

So we investigate the consequences of this embedding from $L$ to $L$ and shall discover that such indiscernibility of these critical points in fact characterizes such embeddings. Kunen [16] showed that if $\exists \bar{j}: L \longrightarrow_{e} L$, then a number of consequences follow.

(i) Then there is such a $j: L \longrightarrow_{e} L$ with $\operatorname{cp}(j)<\omega_{1}$. Moreover, defining $U_{0}$ from such a $j$ with critical point $\kappa_{0}$ least, we are guaranteed well-foundedness of iterated ultrapowers: that is, we may define $j_{01}: L \longrightarrow_{e} L$ by taking the ultrapower of $L$ by $U_{0}$; define $U_{1}$ on $\mathcal{P}\left(\kappa_{1}\right)^{L}$ where $\kappa_{1}={ }_{\mathrm{df}} j_{01}\left(\kappa_{0}\right)$, and then $\operatorname{Ult}\left(L, U_{1}\right)$ will also be well founded. We then may transitivize it and obtain $L$ again. We thus have an embedding map $j_{12}: L \longrightarrow_{e} L$ with critical point $\kappa_{1}$; we then define $\kappa_{2}=j_{12}\left(\kappa_{1}\right)$ and $U_{1}$ on $\mathcal{P}\left(\kappa_{2}\right)^{L}$. The process may be iterated without breaking down, forming a directed system $\left\langle\left\langle N_{\alpha}\right\rangle, j_{\alpha \beta}, \kappa_{a}, U_{\alpha}\right\rangle_{\alpha \leq \beta \in \text { On }}$ with (in this case) all $N_{\alpha}=L$ and elementary maps into direct limits at limit stages $\lambda$, and the $\kappa_{\alpha}$ forming a class $C$ of $L$-inaccessibles, which is closed and unbounded below any uncountable cardinal. 
(ii) The iteration points of such ultrapowers enjoy full-blooded indiscernibility properties in $L$ : if $\varphi\left(v_{0}, \ldots, v_{n}\right)$ is any formula of $\mathscr{L}$ and $\vec{\gamma}, \vec{\delta}$ any two ascending sequences from $[C]^{n+1}$, then $(\varphi(\vec{\gamma}) \leftrightarrow \varphi(\vec{\delta}))^{L}$.

Definition 2.6 (The $0^{\#}$-mouse) Let $j_{\alpha \beta}$, and so forth, be as above. Let

$$
M_{0}=\left\langle L_{\kappa_{0}^{+L}}, \in, U_{0}\right\rangle .
$$

This structure is called the $0^{\#}$-mouse, which itself has iterated ultrapowers using maps that are the restrictions of the

$$
j_{\alpha \beta} \uparrow M_{\alpha}: M_{\alpha} \longrightarrow_{e} M_{\beta} \quad \text { where } M_{\alpha}=\left\langle L_{\kappa_{\alpha}^{+L}}, \in, U_{\alpha}\right\rangle .
$$

Remark 2.7 The viewpoint is shifted to that of the mouse $\left(M_{0}\right)$ generating the model (in this case $L$ ). All of this is a paradigm for generalized constructible inner models $K$-the core models.

By these means we argue for the following.

$$
\text { Theorem } 2.8 \text { (ZF) If } \exists j: L \longrightarrow_{e} L \text {, then } \forall \gamma>\omega\left((\gamma \in \text { Card }) \longrightarrow(\gamma \text { Inacc })^{L}\right) \text {. }
$$

Proof The above implies that $C \cap \gamma$ is unbounded below $\gamma$. However, $C$ is closed, so $\gamma \in C$. Each $\gamma \in C$ is inaccessible in $L$.

Theorem 2.9 (Jensen) (ZF) Suppose $\gamma \in \operatorname{SingCard,~} \gamma \geq \omega_{2}$, but $(\gamma \in \operatorname{Reg})^{L}$. Then $\exists j: L \longrightarrow_{e} L$, with $j \neq \mathrm{id}$; that is, $0^{\#}$ exists.

Proof Suppose $\neg \exists j: L \longrightarrow_{e} L$, but $\gamma$ is chosen least with $\gamma \in$ SingCard but $(\gamma \in \operatorname{Reg})^{L}$. Without loss of interest, we shall assume that (i) $\operatorname{cf}(\gamma)>\omega$; (ii) $\delta<\gamma \longrightarrow \delta^{\omega}<\gamma$. Let $\tau=\operatorname{cf}(\gamma)$. By assumption then $\tau<\gamma$, and so we may choose $X_{0} \subseteq \gamma$ with $\left|X_{0}\right|=\tau$ but $X_{0}$ unbounded in $\gamma$. By (ii) we will assume also that for some $X \supset X_{0}$ we have (a) $\gamma \in X \prec L_{\gamma+L}$ (b) ${ }^{\omega} X \subseteq X$ (c) $|X|=\tau^{\omega}<\gamma$.

Let $\pi:\langle X, \in\rangle \longrightarrow\langle M, \in\rangle=\left\langle L_{\bar{\delta}}, \in\right\rangle$ be the collapsing isomorphism with $\pi(\gamma)=\delta$, say. Then we have

(1) $\operatorname{cf}(\delta)=\tau$ also, with $|\bar{\delta}|=|M|=|X|<\gamma$.

Suppose that we had $\mathcal{P}(\delta)^{M}=\mathcal{P}(\delta)^{L}$. Then we could define a measure derived from $\pi^{-1}$ in the usual manner: let $\alpha=\operatorname{crit}\left(\pi^{-1}\right)$, and define $U$ by

$$
Z \in U \Longleftrightarrow Z \in \mathcal{P}(\delta)^{M} \wedge \alpha \in \pi^{-1}(X) .
$$

Then $U$ would be a countably complete ultrafilter on $\mathcal{P}(\delta)^{L}$ (that is why we chose ${ }^{\omega} X \subseteq X$ as this implies $\left.{ }^{\omega} M \subseteq M\right)$, and this in turn implies that $\operatorname{Ult}(L, U)$ is well founded. However that implies $\exists j: L \longrightarrow_{e} L$ :

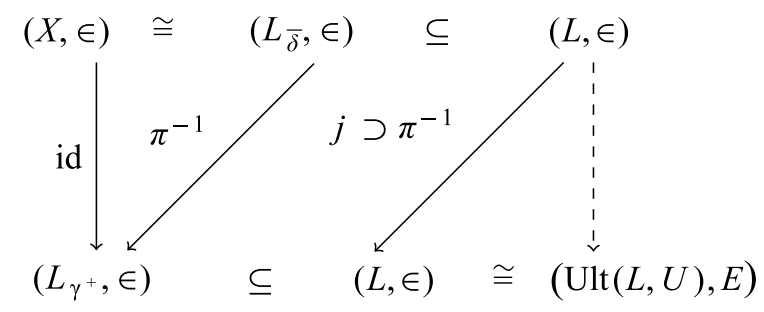


Hence we must have $\mathcal{P}(\delta)^{M} \subsetneq \mathcal{P}(\delta)^{L}$. So

(2) $\exists \beta \geq \bar{\delta}\left(\operatorname{Def}\left(L_{\beta}\right) \cap \mathcal{P}(\delta)\right) \nsubseteq L_{\bar{\delta}}$.

Choose $\beta$ least so that (2) holds. By so-called fine structural methods Jensen showed how there is a superstructure $L_{\eta}$ for some $\eta>\gamma^{+L}$ and a sufficiently elementary map $\tilde{\pi} \supset \pi^{-1}, \tilde{\pi}: L_{\beta} \longrightarrow L_{\eta}$, and because there is a "new" subset of $\delta$ definable over $L_{\beta}$ there must also be a "new" subset of $\gamma=\pi^{-1}(\delta)$ that is not in $L_{\eta}$. However this is absurd as by $L$ 's construction $\left(\mathcal{P}(\gamma) \subset L_{\gamma^{+}}\right)^{L}$.

Remark 2.10 The assumptions (i) and (ii) can be dropped, but not without some difficulty, in particular when $\operatorname{cf}(\gamma)=\omega$; however the format of the argument remains roughly the same.

Theorem 2.11 (Jensen: The full $\boldsymbol{L}$-covering lemma) $\left(\mathrm{ZF}+\neg \mathbf{0}^{\#}\right) \quad$ For any $X \subseteq$ On, if $|X|>\omega$, then there exists $Y \in L$ with (a) $|Y|=|X|$ and (b) $Y \supseteq X$.

The above result Theorem 2.9 is then a corollary of this. As are the following.

Corollary $2.12\left(\mathrm{ZF}+\neg 0^{\#}\right.$ exists)

(a) Let $(\tau \in \operatorname{Reg})^{L}$ with $\tau \geq \omega_{2}$. Then $\operatorname{cf}(\tau)=|\tau|$.

(b) Let $\tau \in$ SingCard. Then $\tau^{+}=\tau^{+L}$.

Remark 2.13 Part (b) of Corollary 2.12 is sometimes called WCL the weak covering lemma. The reason is that for other inner models $M$ we may have WCL $(M)$ provable (obtained by replacing $L$ by $M$ in the corollary's statements), while the full $\mathrm{CL}(M)$ is not. There is a result of Mitchell that shows that if there are sufficiently many measurable cardinals in an inner model, there may be many so-called Prikry sequences (generic for Prikry forcings associated with the measurables) in $V$ that must mess up the full covering lemma.

\section{Generalizations}

If $0^{\#}=M_{0}$ exists as above, perhaps there is no nontrivial $j: L\left[0^{\#}\right] \longrightarrow_{e} L\left[0^{\#}\right]$, and then we have a $\mathrm{CL}\left(L\left[0^{\#}\right]\right)$ ? This is indeed the case; however, if this new assumption fails, then we have $\left(0^{\#}\right)^{\#}$. We then get a theorem along the lines of a new covering lemma in the form of $\mathrm{CL}\left(L\left[0^{\#}\right]\right)$ which holds if and only if $\neg j: L\left[0^{\#}\right] \longrightarrow_{e} L\left[0^{\#}\right]$. $\left(0^{\#}\right)^{\#}$ is again a countable object, and we can repeat this process. After we have done this uncountably often our \#-like mouse objects are no longer countable and we have to resort to uncountable mice $M$.

Instead of toiling inductively through seas of such objects, Dodd and Jensen first proved the following.

Theorem 2.14 (Dodd and Jensen [5]) (ZF) There is an inner model $K^{\mathrm{DJ}}$, so that if there is no inner model with a measurable cardinal, then (a) there is no nontrivial embedding $j ; K^{\mathrm{DJ}} \longrightarrow_{e} K^{\mathrm{DJ}}$ and $(b) \mathrm{CL}\left(K^{\mathrm{DJ}}\right)$.

This was the first core model to go beyond $L$ (if one discounts the models $L\left[0^{\#}\right]$, etc.).

Theorem 2.15 (Steel [35]) (ZFC) If there is no inner model for a Woodin cardinal, then there is a model $K^{\text {Steel }}$, which is again rigid, and over which $\mathrm{WCL}\left(K^{\mathrm{Steel}}\right)$ holds.

Perhaps there is some ultimate model which, although not $L$, is an inner model, $L[E]$, say, which is the "core" of $V$, with $E$ coding up all possible "measures" or means of generating embeddings of models, and hopefully one has, say, $\operatorname{WCL}(L[E])$ ? Maybe, 
but to date the following "inner model program" has worked on the following inductive template. Assume that you have built core models $K^{\Gamma^{\prime}}$ for any strictly weaker large cardinal assumption $\Gamma^{\prime}$ than "There is an inner model with a large cardinal $\Gamma$." Now assume that there is no inner model for such a cardinal as $\Gamma$, and build $K^{\Gamma}$, which, you should show, still under this assumption, is rigid and over which WCL $\left(K^{\Gamma}\right)$ holds. Finally, if the latter fails you have (by contraposition) an inner model with a $\Gamma$-cardinal. Continue. This programmatic approach to filling out $V$ with wider and thicker inner models depending on the strength of the large cardinals existing in inner models of $V$ has become known as the "inner model program." Currently, however, the program is stuck at the level roughly of $\Gamma$ a Woodin limit of Woodin cardinals.

We have further remarks.

Theorem 2.16 (Magidor [18]) (ZF) Assume $\neg 0^{\#}$. If $X \subseteq$ On is uncountable and closed under the primitive recursive set functions, then $X=\bigcup_{n<\omega} Y_{n}$ with each $Y_{n} \in L$.

This is thus a decomposition rather than a covering theorem, but its proof is essentially (but perhaps surprisingly) a variant on Jensen's argument. The primitive recursive set functions mentioned are a mild collection of absolute functions on sets generalizing those on numbers (cf. Devlin [3]).

For many constructions, ordinal combinatorial properties up to a successor cardinal $\gamma^{+}$are needed. The significance of the WCL over a model $M$, say, is that $\gamma^{+}=\gamma^{+M}$ for certain cardinals of $V$ (including the singular cardinals). The requisite ordinal combinatorial property may be established in the inner model $M$. Providing that the property is then sufficiently simple that it is absolute to $V$, we then have that the combinatorial property is valid in $V$ as well and is available for us for that original construction. The weak covering lemma (under the rigidity assumption as always) has thus delivered for us a piece of absolute knowledge about $V$ : one such combinatorial principle is the following.

$\square_{\gamma}$ : There is $\left\langle C_{\alpha} \mid \alpha \in \operatorname{Sing} \cap \gamma^{+}\right\rangle$with

(i) $C_{\alpha} \subseteq \alpha$ is closed; it is unbounded in $\alpha$ if $\operatorname{cf}(\alpha)>\omega$;

(ii) $\operatorname{otp}\left(C_{\alpha}\right)<\alpha$;

(iii) $\beta \in C_{\alpha} \longrightarrow C_{\beta}=\beta \cap C_{\alpha}$.

Such a sequence can be thought of as a uniformly presented sequence of witnesses to the singularity of ordinals below $\gamma^{+}$, which cohere or glue together nicely as expressed in (iii). This is useful for inductive constrictions up to $\gamma^{+}$and, in particular, for singular $\gamma$. Since the properties (i)-(iii) are absolute between $M$ and $V$, if such can be constructed in $M$ and $\gamma^{+}=\gamma^{+M}$ we have such a good sequence in $V$.

Remark 2.17 Jensen first established $\square_{\gamma}$ for all $\gamma \in$ Card, in $L$. This was then established in the core models over the years as they were developed (in $K^{\mathrm{DJ}}$ by the author, in $K^{\text {Steel }}$ by Schimmerling and Zeman [30], and with other results by other set theorists for intermediate models which we have not defined; see also Schimmerling and Zeman [29] for an overview). As intimated, principles such as $\square_{\gamma}$ can be seen as identifying very uniform singularizing functions for singular ordinals. Certain large cardinals have an effect of implying the nonexistence of certain such sequences in $V$. Other forcing axioms outright contradict the existence of any $\square_{\gamma}$-sequences at all. The study of such principles and their various weakenings has told us a lot about the 
interrelationship between large cardinals and inner models. The reference [29] gives an outline of which inner models can admit such sequences: it was a fundamental question which these authors solved as to which canonical inner models could in the end support such sequences. Since it was known that large cardinals contradicted $\square$-principles, it was germane to find precisely where these principles broke down.

Remark 2.18 It is possible to establish the "correct successor cardinal" computation for certain classes of regular limit cardinals $\gamma$ other than singulars alone: for $\gamma$ weakly compact in $V$ (Jensen originally for $L$ ); $\gamma$ Ramsey (see Mitchell [24] originally for $K^{\mathrm{DJ}}$, later in Mitchell [25] for $\left.K^{\text {Steel }}\right) ; \gamma$ Jonsson (Welch [37] for $K^{\text {Steel }}$ ), again thereby showing that for cardinals in these classes, their successors in $V$ also enjoy certain combinatorial properties. However for inaccessibles in general this will fail.

\section{Determinacy: The Martin-Harrington Theorem}

We have mentioned extremely briefly the history of determinacy; a complete source for this material is Moschovakis [26]. We have stated that the regularity properties hold for the projective sets, if all such sets are determined. In this section we define the basic terms relating to determinacy and outline the route leading from large cardinals to projective determinacy. In particular, we demonstrate the key concepts of weakly homogeneous tree and the (weakly) homogeneously Suslin property for sets of reals (see Definitions 3.11, 3.14 below). It is the descriptive set-theoretical analysis and representation of sets via trees that goes back implicitly to Suslin for $\Sigma_{1}^{1}$-sets and, for trees with special measures on them, that enables these theorems. The large cardinals enter the picture, as they are needed to provide the special measurability properties on the trees to make the analysis work. However, it was then seen later that the determinacy of sets which became thus provable in turn proved "reversals" in the form of demonstrations of the existence of inner models of the large cardinals which had been needed to prove the determinacy in the first place. (Notice that determinacy statements alone, being as they are statements about the existence of strategies, which are essentially themselves reals and so statements about elements of $V_{\omega+1}$, cannot prove that large cardinals actually exist in $V$ as the latter statements are about, say, some large $V_{\kappa+1}$, but they can, and do, prove the existence of inner models of those large cardinals.) The relationship between the determinacy of coanalytic sets and embeddings of $L$ was thus the first evidence of a very rich picture that subsequently emerged. What was at first dark and mysterious about determinacy became fully understood through this analysis. Whatever one's position on the concept of large cardinals or strong axioms of infinity, we cannot understand determinacy of the projective sets, say, without it. The "reversals" alluded to above prove this. Even if one dislikes arguments from "extrinsic evidence" to the existence of large cardinals as actual sets, there is no denying their explanatory, and so instrumental, power. Probably for many set theorists that instrumental power alone is a considerable motive for their adoption. Explanatory power is a strong conceptual motivator in other areas of mathematics: mathematicians would have no hesitation in embedding what they were doing in some larger or new kind of space, if it led to understanding. If we regard set theory purely as a mathematical activity, for a moment imagining it shorn of any foundational pretensions, then adoption of such axioms might well be considered unexceptionable. 
Another significant definability property one may ask for is uniformization. Suppose that $\Gamma, \mathrm{H}$ are two point classes of sets of reals. We say that $\operatorname{Unif}(\Gamma, \mathrm{H})$ holds if whenever $Q \in \Gamma \cap \mathcal{P}\left({ }^{k} \mathbb{R} \times \mathbb{R}\right)(k<\omega)$, then there is $P \subseteq Q, P \in \mathrm{H}$, and

$$
\forall x[\exists y Q(y, x) \Longrightarrow \exists ! y P(y, x)] .
$$

( $P$ thus acts as a function uniformizing the relation $Q$ by choosing a unique element in the relation for each $x$ for which such is possible.) Classically one had (the Novikoff-Kondo-Addison theorem) Unif $\left(\Pi_{1}^{1}, \Pi_{1}^{1}\right)$. We let PU (projective uniformization) abbreviate the statement that every projective relation can be projectively uniformized. It is the fundamental outcome of Moschovakis's analysis of the scale property in the projective hierarchy that projective determinacy implies the existence of projectively definable scales, and thence that PU holds (again, cf. [26]).

At a first encounter it seems an unusual position to take, to posit winning strategies for infinite games as plausible set-theoretical axioms. Why should such strategies even exist? However we see now that the regularity properties of sets is actually equivalent to determinacy, that is, to the existence of such strategies, for all sets definable from $\mathbb{R}$ and the ordinals (cf. Theorem 1.9). The regularity properties have been the bread and butter of analysts for decades and are innately uncontroversial. That the early analysts were unable to establish these properties beyond the coanalytic, was indeed because they were trammeled by working, albeit implicitly, only with ZFC-provable tools.

\section{Games}

Definition 3.1 For $A \subseteq \omega^{\omega}$ (or more generally $X^{\omega}$ ) the infinite perfect information game $G_{A}$ is defined between two players $I, I I$, alternately playing elements $n_{i}, m_{i} \in X$ :

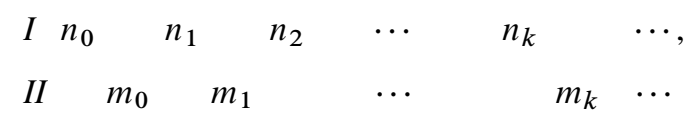

together construed as constructing $x=\left(n_{0}, m_{0}, \ldots, n_{k}, m_{k}, \ldots\right) \in X^{\omega}$.

We say that $I$ wins if and only if $x \in A$; otherwise $I I$ wins. Notions of strategy and winning strategy are defined in the obvious fashion. Notice that for $A \subseteq \omega^{\omega}$ a strategy $\sigma$ for, for example, $I$ is a map from $\bigcup_{k}{ }^{2 k} \omega \longrightarrow \omega$. Since there is a recursive bijection ${ }^{<\omega} \omega \leftrightarrow \omega$, we can think of $\sigma$ as essentially a subset of $\omega$ or, again, as a real number.

We speak of a topology of open and closed sets of a space $X^{\omega}$ by letting a typical basic open set be a neighborhood $N_{s}$ where $s \in \mathrm{Seq}_{X}$ is a finite sequence of elements of $X$ :

$$
N_{s}={ }_{\mathrm{df}}\left\{x \in X^{\omega} \mid \exists k<\omega(x \uparrow k=s\} .\right.
$$

Notice that this is suitable for talking about payoff sets in such games: if in the above $A$ is an $N_{s}$, then essentially if $I$ is to win, then he will have done so after $|s|$ many rounds of the game (where $|s|$ is the length of the sequence $s$ ). An open set is then a countable union of basic open neighborhoods; a closed set is a complement of an open set, and so forth. When $X=\omega$ and an open set, $U$, is given as a union of basic open neighborhoods defined by a recursively given set of sequences numbers $s$ coding elements of ${ }^{<\omega} \omega$, then we say that $U$ is semirecursive, or $\Sigma_{1}^{0}$. More generally we speak of open sets and $\Sigma_{\mathbf{1}}^{\mathbf{0}}$-sets in parameter codes which are themselves subsets 
of $\omega$ or again real numbers. If $A$ is an open set in this topology, then $I$ will win by essentially winning at some finite stage of the game; for $I I$ to win, then she or he must be vigilant to the end to play into the complementary closed set.

The basic theorem here is the following.

Theorem 3.2 (Gale and Stewart) (ZF) Let $A \subseteq X^{\omega}$ be an open set. Then $G_{A}$ is determined; that is, one of the players has a winning strategy.

Proof Note that if $I$ wins it is because the play has been maneuvered so that there is a finite stage $\left(n_{0}, m_{0}, \ldots, n_{k}\right)$ so that $N_{\left(n_{0}, m_{0}, \ldots, n_{k}\right)} \subseteq A$. Essentially $I$ has won by this stage as it matters not what $n_{l}$ is played for $l>k$. Player $I I$, however, playing into the closed set which is $X^{\omega} \backslash A$, must be vigilant to the end in order to win. Suppose then that $I$ has no winning strategy. Then for every $n_{0}$ $I I$ has a reply $m_{0}$ so that $I$ has no winning strategy in the game $G_{A /\left(n_{0}, m_{0}\right)}$, where $A /\left(n_{0}, m_{0}\right)=\mathrm{df}_{\mathrm{df}}\left\{x \in A \mid x(0)=n_{0}, x(1)=m_{0}\right\}$. For, if there was an $n_{0}$ such that $I$ did always have a winning strategy in this latter game for whichever $m_{0} I I$ played, $\sigma\left(m_{0}\right)$, say, then this would amount to a winning strategy for $I$ in $G_{A}$ : first play $n_{0}$, wait for $m_{0}$, and then use $\sigma\left(m_{0}\right)$. Thus given $n_{0} I I$ should play $m_{0}$ so that $I$ has no such strategy. However if she or he continues in this way, this is a winning strategy for $I I$ : always respond so that $I$ has no winning strategy from that point on. The resulting play $x$ cannot be in $A$.

The maxim here is that we can always in ZFC prove Det(Open) for any space $X^{\omega}$ and so by taking complements Det(Closed) too. (AC is needed only to well order $X$ if need be.) Many proofs of determinacy of complicated sets in $\omega^{\omega}$ or $X^{\omega}$ involve reducing the game to a closed game in some larger space $Y^{\omega}$. The latter are determined by the Gale-Stewart theorem. The difficulty arises in showing that the player with the winning strategy for the open set on the space $Y^{\omega}$ also has one for the related but much more complicated set in $X^{\omega}$.

\section{Definition 3.3}

(i) A tree $T$ on $\omega \times X$ (for $X \neq \varnothing$ ) is a set of sequences in $\bigcup_{k}{ }^{k} \omega \times{ }^{k} X$, where if $(\sigma, u)$ and $k \leq|\sigma|=|u|$, then $(\sigma \uparrow k, u \uparrow k) \in T$. Similarly define trees on ${ }^{n} \omega \times X$.

(ii) For such a tree $T$ we set

$$
T_{\sigma}={ }_{\mathrm{df}}\{u \mid(\sigma, u) \in T\}, \quad T_{\sigma}^{\subseteq}={ }_{\mathrm{df}} \bigcup_{k \leq|\sigma|} T_{\sigma \uparrow k}, \quad \text { and } \quad T_{x}=\bigcup_{k} T_{x \uparrow k} .
$$

\section{Definition 3.4 For $T$ a tree,}

(i) $[T]={ }_{\mathrm{df}}\{(x, f) \mid \forall k(x \uparrow k, f \uparrow k) \in T\}$ is the set of branches through $T$;

(ii) $p[T]={ }_{\mathrm{df}}\left\{x \in{ }^{\omega} \omega\left(\operatorname{or}^{k}\left({ }^{\omega} \omega\right)\right) \mid \exists f(x, f) \in[T]\right\}$ is the projection of $T$;

(iii) a set $A \subseteq{ }^{k}\left({ }^{\omega} \omega\right)$ is $\kappa$-Suslin (for $\kappa \geq \omega, \kappa \in$ Card) if $A=p[T]$ for some tree on $\omega \times \kappa$.

Clearly, a tree is well founded (under $\supseteq$ ) if $[T]=\varnothing$. For a $C \subseteq \omega \times X$ a closed set, there is a tree $T$ on $\omega \times X$ with $C=[T]$. This is true in particular for $X=\omega$. If $C$ has a recursively open complement, then we may take $T$ as recursive set of sequences from $\bigcup_{k}\left({ }^{k} \omega \times{ }^{k} \omega\right)$.

If $A \subseteq \omega^{\omega}$ is $\Sigma_{1}^{1}$, then $A=p[T]$ for a tree on $\omega \times \omega$-thus such sets are projections of closed sets-and conforms to the idea that $x \in A \leftrightarrow \exists y \in \omega^{\omega}(x, y) \in[T]$. 
This classical result (due to Suslin) is sometimes stated that analytic $\left(=\boldsymbol{\Sigma}_{\mathbf{1}}^{\mathbf{1}}\right)$ sets are " $\omega$-Suslin." Many of the classical properties of analytic sets as studied by analysts can be attributed to this (and similar) representations.

Given this last fact, we can represent $\boldsymbol{\Pi}_{\mathbf{1}}^{\mathbf{1}}$-sets, being complements of $\boldsymbol{\Sigma}_{\mathbf{1}}^{\mathbf{1}}$ 's, as those sets where the tree $T$ for the complement is well founded. We may thus define rank functions for such trees by finding functions that map the nodes into the ordinals in a tree-order-preserving way. We do this next.

Let then $A \in \Pi_{1}^{1}$ be a (lightface) coanalytic set. Then by the above discussion there is a recursive tree $T$ (meaning that there is a recursive set of sequence numbers) on $\omega \times \omega$ with

$$
\forall x\left(x \in A \leftrightarrow T_{x} \text { is well founded }\right) .
$$

$T_{x}$ being countable, we have that $\operatorname{rk}\left(T_{x}\right)<\omega_{1}$. Consequently,

$$
x \in A \Leftrightarrow \exists g\left(g: T_{x} \longrightarrow \omega_{1}\right. \text { in an order-preserving way). }
$$

That is, we may define a tree $\hat{T}$ on $\omega \times \omega_{1}$ as follows:

$$
\hat{T}=\left\{(\tau, u)|\forall i, j<| \tau \mid: \tau_{i} \supset \tau_{j} \wedge\left(\tau \uparrow\left|\tau_{i}\right|, \tau_{i}\right) \in T \longrightarrow u(i)<u(j)\right\} .
$$

Then one can see that

$$
x \in A \leftrightarrow \exists g \in{ }^{\omega} \omega_{1}((x, g) \in[\hat{T}]) \leftrightarrow x \in p[\hat{T}] .
$$

The above reasoning thus shows that any $\Pi_{1}^{1}$-set $A$ is $\omega_{1}$-Suslin. Further:

(i) $\hat{T} \in L$, and by the absoluteness of well-founded relations on $\omega$ between $\mathrm{ZF}^{-}$-models containing all countable ordinals $[\hat{T}] \neq \varnothing \leftrightarrow([\hat{T}] \neq \varnothing)^{L}$;

(ii) if the underlying set is a $\Pi_{1}^{1}(a)$-set for some real parameter $a$, then $\hat{T} \in L[a]$. Now the argument can be stepped up to $\Sigma_{2}^{1}$-sets: suppose $x \in B \leftrightarrow \exists y(x, y) \in A$ (we write $B=p A$ ) with $A \in \Pi_{1}^{1}$; hence there is a tree $\hat{T}_{A}$ on $(\omega \times \omega) \times \omega_{1}$ with

$$
(x, y) \in A \leftrightarrow \exists g \in{ }^{\omega} \omega_{1}\left(((x, y), g) \in\left[\widehat{T}_{A}\right]\right) .
$$

However, there is a $\Delta_{0}^{\mathrm{ZF}}$-definable bijection $\omega \times\left(\omega \times \omega_{1}\right) \leftrightarrow(\omega \times \omega) \times \omega_{1}$ thus redefining the tree $\widehat{T}_{A}$ as $\bar{T}$ but on different sequences, so that for any $x, y$ we have $(x, y) \in p\left[\widehat{T}_{A}\right] \leftrightarrow \exists g \in{ }^{\omega} \omega_{1}\left(((x, y), g) \in\left[\widehat{T}_{A}\right]\right) \leftrightarrow \exists g \in{ }^{\omega} \omega_{1}((x,(y, g)) \in[\bar{T}])$. However, now we again use a $\Delta_{0}^{\mathrm{ZF}}$-bijection $\omega \times \omega_{1} \leftrightarrow \omega_{1}$ to recast $\bar{T}$ as a tree $\overline{\bar{T}}$ on $\omega_{1}$ alone; then

$$
x \in B \leftrightarrow x \in p A \leftrightarrow x \in p[\overline{\bar{T}}] .
$$

We have the following.

Theorem 3.5 (Shoenfield) (ZF) Any $\Sigma_{2}^{1}$-set is $\omega_{1}$-Suslin, as a projection of a tree $T \in L$.

Corollary 3.6 (ZF) Let $B$ be any $\Sigma_{2}^{1}$-relation. Then $\exists x B(x) \leftrightarrow(\exists x B(x))^{L}$. In particular, $\Sigma_{2}^{1}$-sentences are absolute between $L$ and the universe $V$. Moreover, if $A \subseteq \mathbb{N}$ is $\Sigma_{2}^{1}$, then $A \in L$.

Theorem 3.7 (Levy) (ZF) There is an ordinal $\sigma_{1}<\omega_{1}^{L}$ such that $\left(L_{\sigma_{1}}, \in\right) \prec \Sigma_{1}$ $(V, \in)$. 
Proof Let $A$ be a $\Sigma_{1}$-sentence in $\mathscr{L}$ true in $V$. By Löwenheim and Skolem, the following holds:

There is a countable well-founded transitive model $(M, \in)$ with $(A)^{M}$.

The latter can be expressed as a $\Sigma_{2}^{1}$-assertion about a real number $x$ (coding such an $(M, \epsilon))$. By Corollary 3.6 there is such an $x$, and $M_{x} \in L_{\omega_{1}^{L}}$. By the upward persistence of $\Sigma_{1}$-sentences $(A)^{M_{x}} \Longrightarrow(A)^{L}$. Hence $L_{\omega_{1}^{L}} \prec \Sigma_{1} V$.

We sometimes wish to be more specific about the tree for $\Pi_{1}^{1}$, and for this we use the Kleene-Brouwer ordering: $<_{\mathrm{KB}}$ is a linear ordering, and it restricts to linear orderings on ${ }^{<\omega} \kappa$ for cardinals $\kappa \geq \omega$. One may check the following:

If $T$ is a tree on $\kappa$, then $T$ is well founded if and only if $T$ is well ordered by $<_{\mathrm{KB}}$. In particular, we have for a $\Pi_{1}^{1}$-set $A$ that there is a tree $T$ on $\omega \times \omega$ so that

$$
x \in A \leftrightarrow T_{x} \text { is well founded } \leftrightarrow T_{x} \text { is well ordered by }<_{\mathrm{KB}} .
$$

For a tree $T$ on $\omega \times \kappa$ we then have a linear ordering $<_{x}$ corresponding to the $\mathrm{KB}$ ordering of $T_{x}$ with the following definition and properties:

$$
\begin{aligned}
i<_{x} j \longleftrightarrow{ }_{\mathrm{df}} & \left(\tau_{i}, \tau_{j} \notin T_{x} \wedge i<j\right) \vee\left(\tau_{i} \notin T_{x} \wedge \tau_{j} \in T_{x}\right) \\
& \vee\left(\tau_{i}, \tau_{j} \in T_{x} \wedge \tau_{i}<_{\mathrm{KB}} \tau_{j}\right) .
\end{aligned}
$$

In fact, $<_{x}$ is the union of orderings given by initial segments of $x$ : for $\tau \in{ }^{<\omega} \omega$ define

$$
\begin{gathered}
i<_{\tau} j \longleftrightarrow \mathrm{df}_{\mathrm{d}} i<j<|\tau| \wedge\left(\tau_{i}, \tau_{j} \notin T_{\tau}^{\subseteq} \wedge i<j\right) \vee\left(\tau_{i} \notin T_{\tau}^{\subseteq} \wedge \tau_{j} \in T_{\tau}^{\subseteq}\right) \\
\vee\left(\tau_{i}, \tau_{j} \in T_{\tau}^{\subseteq} \wedge \tau_{i}<_{\mathrm{KB}} \tau_{j}\right) .
\end{gathered}
$$

Then

(i) $<_{x}=\bigcup_{k}<_{x \uparrow k}$;

(ii) define $T_{A}^{*}$ on $\omega \times \kappa$ by

$$
(\tau, u) \in T_{A}^{*} \longleftrightarrow \mathrm{df}_{\mathrm{df}} \forall i, j<|\tau|\left(i<_{\tau} j \leftrightarrow u(i)<u(j)\right) .
$$

Then

$$
x \in A \leftrightarrow \exists g \in{ }^{\omega} \kappa\left((x, g) \in\left[T_{A}^{*}\right]\right) .
$$

In the above, $\kappa \geq \omega$ may be any cardinal of course. After the basic definitions were introduced $\operatorname{Det}\left(\Sigma_{3}^{0}\right)$ (see Davis [2]) was the extent of provable determinacy (indeed provable within $Z_{2}$ ). Remarkably Friedman [6] showed that for Borel determinacy roughly, order type $\alpha$-iterations of the power set operation (together with some replacement) would be needed to establish $\operatorname{Det}\left(\Sigma_{\alpha}^{0}\right)$, thus establishing that $Z \nvdash \operatorname{Det}\left(\Delta_{1}^{1}\right)$. This phenomenon would start at $\Sigma_{4}^{0}$. Paris then $\operatorname{showed} \operatorname{Det}\left(\Sigma_{4}^{0}\right)$ (using ZFC), but there matters languished.

Theorem 3.8 (Martin [20]) (ZF) We have Det(Borel) for Borel subsets of $\omega^{\omega}$.

\section{Theorem 3.9 (Martin and Harrington) (ZF)}

$$
\exists j: L \longrightarrow_{e} L, \quad j \neq \mathrm{id} \Longleftrightarrow \operatorname{Det}\left(\Pi_{1}^{1}\right) .
$$

Proof $(\longrightarrow)$ (Martin) We assume the left-hand side, and then by the work of Silver and Kunen, we have a closed and unbounded set $C \subseteq \omega_{1}$ of indiscernibles for $(L, \in)$.

Let $A \in \Pi_{1}^{1}$. The usual game $G_{A}$ involves integer moves but has a complicated payoff set. We replace the space $\omega^{\omega}$ with a larger one $X^{\omega}$ for some $X$ to be defined, 
and we relate $A$ to some closed $A^{*} \subseteq X^{\omega}$. By the Gale-Stewart theorem, this is determined. We have to show that winning strategies in $G_{A^{*}}$ can be translated to winning strategies for the same player in $G_{A}$.

Definition 3.10 $G_{A^{*}}$ is defined between two players $I$, II playing as follows:

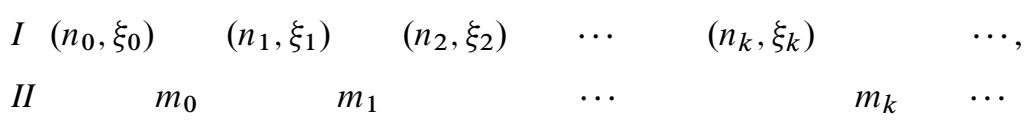

together constructing

$$
x=\left(\left(n_{0}, \xi_{0}\right), m_{0},\left(n_{1}, \xi_{1}\right), m_{1},\left(n_{2}, \xi_{2}\right), m_{2}, \cdots\left(n_{k}, \xi_{k}\right), \ldots\right) .
$$

The rules are that $n_{i}, m_{i} \in \omega, \xi_{i} \in \omega_{1}$. We think of the $\xi_{i}$ as laying out a function $g: \omega \longrightarrow \omega_{1}$ with $g(i)=\xi_{i}$, and the integers yielding $x=\left(n_{0}, m_{0}, n_{1}, \ldots\right)$.

Winning conditions: I wins if and only if $(x, g) \in T_{A}^{*}$.

Then $g$ witnesses that $T_{x}$ is well founded by giving an order-preserving map from $<_{x}$ into $\omega_{1}$. Note that the game is closed in the sense that if $I$ loses it happens at some finite stage (by messing up the ordering, which cannot be redeemed at a later stage). Equivalently, II is playing into an open set, and if she or he wins, does so at a finite round. Note that $T_{A}^{*}$ is defined in $L$, and so, by the Gale-Stewart theorem, is determined in $L$.

Claim If $\sigma^{*}$ is a winning strategy for I (resp., II) in $G_{A^{*}}$ in $L$, then there is a winning strategy for I (resp., II) in $G_{A}$.

Proof of Claim. If $\sigma^{*}$ is a winning strategy for $I$ it can be used to play in $G_{A}$ by suppressing the ordinal moves and clearly wins (both in $L$ and $V$ ). Suppose then that $\sigma^{*} \in L$ is, in $L$, a winning strategy for $I I$.

The idea is that $I I$ simulates a run of $G_{A^{*}}$ by using indiscernibles from $C_{0}={ }_{\mathrm{df}} C \cap \omega_{1}$ as "typical" ordinal moves for $I$. Player $I I$ defines a strategy $\sigma: \bigcup_{n}{ }^{2 n+1} \omega \longrightarrow \omega$ as follows; fix $n<\omega$, and consider the formula $\varphi\left(\omega_{1}, T^{*}, \sigma^{*}, \tau, \vec{\xi}, m\right)$ which defines a term $t\left(\omega_{1}, T^{*}, \sigma^{*}, \tau, \vec{\xi}\right)=m$ :

$$
\vec{\xi} \in{ }^{n+1} \omega_{1} \wedge \sigma^{*}(\tau \uparrow n+1, \vec{\xi}) \in T^{*} \wedge \sigma^{*}(\tau, \vec{\xi})=m .
$$

As $\sigma^{*}, T^{*} \in L$ the term $t$ will have a fixed value $m \in \mathbb{N}$ for any $\vec{\xi} \in{ }^{n+1} C_{0}$ that is chosen, since the latter are indiscernibles for the formula $\varphi$. So she or he sets (in $V$ where $C_{0}$ lives):

$$
\sigma(\tau)=m=t\left(\omega_{1}, T^{*}, \sigma^{*}, \tau, \vec{\xi}\right) \text { for any } \vec{\xi} \in{ }^{n+1} C_{0}
$$

Now argue that $x \in A$, even though II followed this strategy; we would have that there is an order-preserving embedding $g:\left(\omega,<_{x}\right) \longrightarrow\left(C_{0},<\right)$ (as $C_{0}$ is uncountable). However, that corresponds to a run of the game $G_{A^{*}}$ (with ordinal moves delivered by $g$ ), where $I I$ has used $\sigma^{*}$, which was supposed to be winning, which is a contradiction!

It is an exercise to show that if a $G_{A}$ for $A \subseteq{ }^{\omega} \omega$ is determined, $A$ is countable or else contains a perfect subset and hence is the size of the continuum. However, in $L$ there is an uncountable $\Pi_{1}^{1}$-set which has no perfect subset. Conclusion: $\operatorname{Det}\left(\Pi_{1}^{1}\right)$ is false in $L$, and so $\mathrm{ZF} \nvdash \operatorname{Det}\left(\Pi_{1}^{1}\right)$. In the above we constructed a winning strategy 
for $I I$ in $V$. However, the statement " $\exists \sigma\left[\sigma\right.$ is a winning strategy for $I I$ in $\left.G_{A}\right]$ " is a strictly $\Sigma_{3}^{1}$-statement, and as such is not in general absolute to $L$.

We should like to establish $\operatorname{Det}\left(\Pi_{n}^{1}\right)$ for other $n>1$, but this requires considerably more ingenuity. The key is to find representations of projective sets as projections of trees enjoying so-called homogeneity properties which entail that there are measures (and more) on the trees. This tree representation was implicit in the proofs of $\operatorname{Det}\left(\Pi_{1}^{1}\right)$ of Martin and in Martin and Solovay [22] where such trees were used to analyze $\Pi_{2}^{1}$-sets. However the notion was only made explicit in Kechris [13]. For the rest of this article we shall work in ZFC unless otherwise stated.

Definition 3.11 (Homogeneous trees and sets) Let $T$ be a tree on $\omega \times X$, and let $\kappa>\omega$ be a cardinal.

(A) Then $T$ is a $\kappa$-homogeneously Suslin tree if and only if $\exists\left\langle U_{\tau}\right| \tau \in$ Seq $\rangle$, where

(i) each $U_{\tau}$ is a $\kappa$-complete measure on $T_{\tau}$;

(ii) for any $\tau \supset \sigma, U_{\tau}$ projects to $U_{\sigma}$ : that is,

$$
u \in U_{\sigma} \leftrightarrow\left\{v \in T_{\tau}: v \uparrow|\sigma| \in u\right\} \in U_{\tau}
$$

(iii) the $U_{\tau}$ form a countably complete tower: if $\sigma_{i} \in$ Seq, $Z_{i} \in U_{\sigma_{i}}$ are such that $i<j \longrightarrow \sigma_{i} \subset \sigma_{j}$, then there is a $g \in{ }^{\omega} X$ so that $\forall i\left(g \uparrow i \in Z_{i}\right)$.

(B) We say that $A \subseteq \omega^{\omega}$ is $\kappa$-homogeneously Suslin if $A=p[T]$ for a $\kappa$ homogeneously Suslin tree T.

Notice that one way to phrase (iii) is to say that if $x \in p[T]$, then $\left\langle U_{x \uparrow i}: i<\omega\right\rangle$ form a countably complete tower. Although the notion had to be isolated, in fact Martin had used essentially the fact that if there exists a measurable cardinal $\kappa$, then any analytic set is $p[T]$ for a $\kappa$-homogenously Suslin tree on $\omega \times \kappa$.

Theorem 3.12 Suppose that $A$ is $\kappa$-homogeneously Suslin for some $\kappa$. Then $G_{A}$ is determined.

Proof Suppose $A=p[T]$ with $T \kappa$-homogeneously Suslin on $\omega \times \lambda($ some $\lambda \geq \kappa)$.

Definition 3.13 $G_{A^{*}}$ is defined between two players I, II playing as follows:

$\begin{array}{lllllll}I & \left(n_{0}, \xi_{0}\right) & \left(n_{1}, \xi_{1}\right) & \left(n_{2}, \xi_{2}\right) & \cdots & \left(n_{k}, \xi_{k}\right) & \\ I I & m_{0} & m_{1} & \cdots & & m_{k} & \cdots,\end{array}$

together constructing

$$
x=\left(\left(n_{0}, \xi_{0}\right), m_{0},\left(n_{1}, \xi_{1}\right), m_{1},\left(n_{2}, \xi_{2}\right), m_{2}, \cdots\right) .
$$

Rules: We have $n_{i}, m_{i} \in \omega, \xi_{i} \in \lambda$. Winning conditions: As before $I$ wins if and only if $(x, g) \in[T]$ where $g(i)=\xi_{i}$.

Again this is a closed game, and so there is a winning strategy for one of the players in $L[T]$. Now to show that $I I$ has a winning strategy in $G_{A}$ if she has a winning strategy $\sigma^{*}$ in $G_{A^{*}}$, we use the $\omega_{1}$-completeness of the measures rather than indiscernibility.

She defines a strategy $\sigma: \bigcup_{n}{ }^{2 n+1} \omega \longrightarrow \omega$ as follows: for $n<\omega$ and then for $\tau \in \in^{2 n+1} \omega, u \in \in^{n+1} \lambda$, define

$$
\bar{\sigma}(\tau, u)=\sigma^{*}(\tau(0), u(0), \ldots, \tau(2 n), u(2 n)) \in \omega .
$$


Define $Z_{\tau, k}={ }_{\mathrm{df}}\{u \in T(\tau \uparrow n+1): \bar{\sigma}(\tau, u)=k\}$. Since $U_{\tau \uparrow n+1}$ is a measure on $T(\tau \uparrow n+1)$, by its $\omega_{1}$-completeness, for precisely one value of $k$ is $Z_{\tau, k} \in U_{\tau \uparrow n+1}$. So let that value of $k, k_{0}$, say, be the response given by the strategy

$\sigma(\tau)=k_{0} \quad$ where $k_{0}$ is the unique value of $k_{0}$ with $Z_{\tau, k_{0}} \in U_{\tau \uparrow n+1}$.

Again we check that $\sigma$ is a winning strategy for $I I$ in $G_{A}$. If $x$ is a result of a play with II using $\sigma$, but nevertheless $x \in A$, then by (iii) in the definition of $\kappa$-homogeneously Suslin $\exists g \in{ }^{\omega} X$, so that for every $n, g \uparrow n+1 \in Z_{x \uparrow 2 n+1, \sigma(x \uparrow 2 n+1)}$. This implies $(x, g) \in[T]$ and is then a losing outcome of the game where $I I$ in fact uses $\sigma^{*}$, thus contradicting that the latter is a winning strategy for $I I$. Hence $x \in A$.

Hence determinacy would follow if we could establish homogeneity properties for trees.

Definition 3.14 $A \subseteq \omega^{\omega}$ is weakly homogeneously Suslin if $A=p B$, where $B \subseteq\left(\omega^{\omega}\right)^{2}$ is homogeneously Suslin.

In fact this is not the official definition of weakly homogeneously Suslin which defines "weakly homogenous trees" and is in terms of towers of measures, but nevertheless has this equivalence. These were also studied by Kechris [13] and Martin.

Weakly homogeneously Suslin sets (being the projections of weakly homogeneous trees) can be thought of as generalizations of analytic sets and are of great interest in their own right: we have seen that if there is a measurable cardinal, then $\Sigma_{2}^{1}$-sets are weakly homogeneously Suslin.

Theorem 3.15 If a set A is weakly homogeneously Suslin, then it has the regularity properties (Lebesgue measurability, the Baire and perfect subset properties).

It thus has the consequences of being determined, without actually being so. Unfortunately, $A$ being weakly homogeneously Suslin does not imply that it is homogeneously Suslin. However, one important feature is that they do have complements defined as projections of trees $\tilde{T}$ with the latter definable from their weakly homogenously tree $T$.

Lemma 3.16 Let $A$ be $p[T]$ with $T$ weakly homogeneously Suslin. Then there is a tree $\tilde{T}$ with $\omega^{\omega} \backslash A=p[\tilde{T}]$.

However, on its own this is no help. The breakthrough was the following theorem.

Theorem 3.17 (Martin and Steel [23]) Suppose that $\lambda$ is a Woodin cardinal and that $T$ is a $\lambda^{+}$weakly homogeneously Suslin tree. Then for $\gamma<\lambda$, the $\tilde{T}$ above is $\gamma$-homogeneously Suslin.

There is thus some trade-off: the completeness of the measures drops. However, we now have the following.

Theorem 3.18 (Martin and Steel) Suppose that $\lambda_{0}$ is a Woodin cardinal and that $\kappa>\lambda_{0}$ is measurable. Then $\operatorname{Det}\left(\Pi_{2}^{1}\right)$. Further, if $\lambda_{n-1}<\lambda_{n-2}<\cdots<\lambda_{0}$ are $n-1$ further Woodin cardinals, then $\operatorname{Det}\left(\Pi_{n+1}^{1}\right)$. Thus

$$
\mathrm{ZFC}+\text { "there exist infinitely many Woodin cardinals" } \vdash \mathrm{PD} \text {. }
$$

Proof Let $A \subseteq \omega^{\omega}$ be $\Pi_{2}^{1}$. Then $A$ is the complement of a $\Sigma_{2}^{1}$-set on $\omega^{\omega}$, which is itself the projection of a $\Pi_{1}^{1}$-set $B \subseteq\left(\omega^{\omega}\right)^{2}$. B is $\kappa$-homogeneously Suslin for some 
homogeneous tree $T$, as $\kappa$ is measurable, and a fortiori is also $\lambda_{0}^{+}$-homogeneously Suslin. By Theorem 3.18 we have a $\gamma$-homogeneously Suslin tree $\tilde{T}$ projecting to $A$ with $\gamma$-complete measures. By Theorem 3.12 we have that $G_{A}$ is determined, and we are done. The last two sentences follow by repetition of the argument.

The exact consistency strength of the assumption above is slightly stronger, with the $(\Longleftarrow)$ being very involved.

Theorem 3.19 (Woodin) We have

$$
\begin{aligned}
& \operatorname{Con}(\mathrm{ZFC}+\text { "there exist infinitely many Woodin cardinals") } \\
& \Longleftrightarrow \mathrm{Con}\left(\mathrm{ZFC}+\mathrm{AD}^{L(\mathbb{R})}\right) .
\end{aligned}
$$

The final conclusion of Theorem 3.18 yields the boundary of the provability of PD from large cardinal axioms. This is not a relative consistency result; PD holds outright. Set forcing can change the character of the universe locally but, roughly speaking, does not destroy large cardinal properties beyond the rank of the partial order. Consequently if $V$ has a proper class of Woodin cardinals, then PD is not only provable outright but is absolute into set-forcing extensions. Much more is possible.

Theorem 3.20 (Woodin) Suppose that there is a proper class of Woodin cardinals; then $\operatorname{Th}(L(\mathbb{R}\})$ is absolute with respect to set forcing. Thus if $V[G]$ is a setgeneric extension, then

$$
(L(\mathbb{R}))^{V} \equiv(L(\mathbb{R}))^{V[G]} .
$$

The larger cardinals that we look at next will again all prove $\mathrm{PD}$ and in fact $\mathrm{AD}^{L(\mathbb{R})}$ outright. We have seemingly reached a particular stage beyond which these determinacy properties are simply unavoidable.

\section{Large Cardinals}

Much large cardinal theory is about which ultrafilters can or do exist on (large) sets; in particular, when those large sets are the power set of some cardinal of an inner model $M$ (which might be $V$ itself), then there is usually an equivalent formulation in terms of elementary embeddings, of that inner model $M$. In the past, large cardinals, or the axioms asserting their existence, were often claimed as justified by a variety of differing arguments (an example being the paper of Solovay, Reinhardt, and Kanamori [34]). Such arguments tended to give a somewhat patchwork view of large cardinals: some justified "by analogy with $\omega$," others by reflection principles of one kind or another. Contemporary set theory now tends to see large cardinals in a linear, indeed well-founded, hierarchy of consistency strength, but moreover as providing a lined-up picture of embedding properties ordered by the degree that the embedding "pulls in" to its range model, more, or less, of the $V_{\alpha}$-hierarchy. There is some oversimplification here, as pulling in some $V_{\lambda}$ into a range model $M$ will be weaker than ensuring that such an $M$ has more closure properties, such as being closed under $\lambda^{\prime}$-sequences for a $\lambda^{\prime}<\lambda$. However, the overall slogan holds true: the more closure properties the range model enjoys, the stronger the embedding and the stronger the large cardinal needed to justify it. Again the unificatory impulse which sees large cardinals as positioned within a somewhat continuous spectrum of embeddability relations can be said to lead to a greater understanding of possible 
embedding phenomena. The very possibility of mathematically defining an embedding of $L$ to $L$ immediately then impresses us with the need to study the whole such hierarchy: if the universe of constructible sets can embed to itself, then why not the model $K^{D J}$ ? However, if this occurs, then there is an inner model with a measurable cardinal (see Theorem 2.14 above). In short, there seem to be no lines to be drawn here.

Such embeddings are almost all expressible in terms of the existence of ultrafilters, or in other words, measures (or sequences of such) on certain sets. We have seen that a measure $U$ on $\mathcal{P}(\kappa)$ in $V$ yields an ultrapower $(\operatorname{Ult}(V, U), E)$ which is well founded and hence isomorphic to a transitive inner model $(M, \in)$ of ZFC. The following facts hold:

- $V_{\kappa+1}=\left(V_{\kappa+1}\right)^{M}$;

- $(j(\kappa) \text { is measurable with measure } j(U))^{M}$;

- $U \notin M$ and thus $V_{\kappa+2} \neq\left(V_{\kappa+2}\right)^{M}$;

- $\kappa$ may, or may not, be measurable in $M$ because of the presence in $M$ of some other measure $\bar{U}$. (If $\kappa$ was the least measurable of $V$, then it cannot contain such a $\bar{U}$, by the Scott argument.)

Thus given an elementary embedding $j: V \longrightarrow_{e} M$, the above shows precisely which initial part of $V$ can be expected to be in $M$ in general, namely, $V_{\kappa+1}$. The first generalizations of this concept consider sequences of such measures concentrating on different subsets of the same cardinal $\kappa$. (There are potentially then $2^{2^{\kappa}}$ of those for a given measurable cardinal $\kappa$.) Beyond that we start to ask for sets of higher rank to be in the range model, and this motivates the next definition.

Definition 4.1 A cardinal $\kappa$ is $\alpha$-strong if there is an embedding $j: V \longrightarrow_{e} M$ with $V_{a}=\left(V_{\alpha}\right)^{M}$, with $\mathrm{cp}(j)=\kappa$, and $j(\kappa) \geq \alpha$.

Thus a measurable cardinal is $(\kappa+1)$-strong. The larger the $\alpha$, the stronger the embedding, as more of the initial $V$ hierarchy is preserved by the identity map into the inner model $M$.

Definition 4.2 A cardinal $\kappa$ is strong if it is $\alpha$-strong for all $\alpha$.

Note the order of quantifiers: for every $\alpha$ there is an embedding $j$, depending on $\alpha$. One may wonder about the first-order formalizability of the above notions in ZF. However just as the first statement of the existence of a measure on $\mathcal{P}(\kappa)$ is equivalent to the existence of a class embedding $j$ (which we might formulate in $\mathrm{ZFC}_{j}$ ), it is possible to give an extender representation of such embeddings. Thus for an $\alpha$-strong embedding $j$ we may find a generalization of a measure on $\mathcal{P}(\kappa)$ called an extender, which we may think of as given by a sequence of measures $\left\langle E_{a}: a \in[\alpha]^{<\omega}\right\rangle$ with each $E_{a}$ itself a measure on $\mathcal{P}\left([\kappa]^{|a|}\right)$.

Given an $\alpha$-strong embedding $j: V \longrightarrow_{e} N$ we define an $\alpha$-extender at $\kappa$ generalizing what we did for measures:

$$
X \in E_{a} \leftrightarrow_{\mathrm{df}} X \in \mathcal{P}\left([\kappa]^{|a|}\right) \wedge a \in j(X) .
$$

The sequence $\mathcal{E}=\left\langle E_{a}: a \in[\alpha]^{<\omega}\right\rangle$ then has satisfactory coherence properties, in fact enough so that we can define an extender ultrapower $(\operatorname{Ult}(V, \mathcal{E}), E)$ from it. In the situation described, this ultrapower has a well-founded $E$-relation and is again 
isomorphic to some $(M, \in)$ which in general is not necessarily equal to $(N, \in)$ but which can be elementarily embedded back into $(N, \in)$, and we thus shall have again

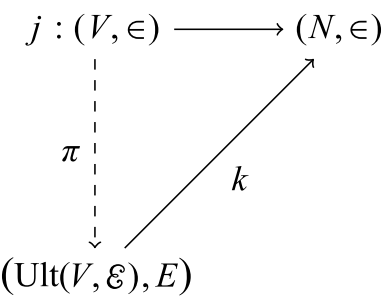

It is possible to view the $\operatorname{Ult}(V, \mathcal{E})$ as a direct limit of the "ordinary" ultrapowers by the measures $E_{a}$. It is part of the flexibility of the approach that this is inessential though.

Having thus generalized the notion of measure ultrapower to extender ultrapower we can use these to give us first-order formulations of $\alpha$-strong, and so forth. A simplified statement is as follows.

Lemma 4.3 Let $\alpha$ be a strong limit cardinal; then $\kappa$ is $\alpha$-strong if and only if there is an $\alpha$-extender sequence $\mathcal{E}=\left\langle E_{a}: a \in[\alpha]^{<\omega}\right\rangle$ at $\kappa$, with $V_{\kappa+\alpha} \subseteq \operatorname{Ult}(V, \mathcal{E}) \wedge$ $j(\kappa)>\alpha$.

The notion of $\kappa$ being strong is then also first-order (although involving a quantifier over On). One may note the following easily proven fact: $\kappa$-strong implies that $V_{\kappa} \prec \Sigma_{2} V$.

Definition 4.4 $\kappa$ is superstrong if there is $j: V \longrightarrow_{e} M$ with $V_{j(\kappa)} \subseteq M$.

Note that $\alpha$-strong only asked for $V_{\alpha} \subseteq M$ whilst $j(\kappa)>\alpha$. This seemingly innocuous extension is in fact a powerful strengthening. Again it has a first-order formalization. We proceed to a definition of Woodin cardinal. First we define a strengthening of the concept of strong.

Definition 4.5 Let $A \subseteq V$. We say that $\kappa$ is $A$-strong in $V$ if for every $\alpha$ there are $\mathrm{M}, B \subseteq V$ with $I M(M)$ and an $\mathscr{L}_{\dot{\epsilon}, \dot{A}}$-elementary embedding

$$
j:\langle V, A\rangle \longrightarrow_{e}\langle M, B\rangle \quad \text { such that } \operatorname{cp}(j)=\kappa, V_{\alpha} \subseteq M \text { and } V_{\alpha} \cap A=V_{\alpha} \cap B .
$$

This is not a first-order formalization, but now consider an inaccessible $\lambda$ and relativize the notion from $V$ down to $V_{\lambda}$.

Theorem 4.6 An inaccessible cardinal $\lambda$ is called Woodin if for every $A \subseteq V_{\lambda}$ there is a $\kappa<\lambda$ which is A-strong in $V_{\lambda}$.

There are many other equivalent formulations (cf. Kanamori [12, Section 26]). A Woodin cardinal is necessarily Mahlo but may fail to be weakly compact. It turned out that this is precisely the right concept to analyze the various determinacy properties. Subsequently it turned out to be also precisely the right concept to gauge a whole host of other set-theoretical phenomena, and thus it has become one of the central notions of modern set theory.

\section{Lemma 4.7}

(i) If $\kappa$ is superstrong, then it is a Woodin limit of Woodins.

(ii) If $\lambda$ is Woodin, then ("there are arbitrarily large strong cardinals") $V_{\lambda}$. 
A particular constellation of cardinals is also of interest for determinacy of infinite games played with reals, rather than integers. The assertion $\mathrm{AD}_{\mathbb{R}}$ is that for every $\mathcal{A} \subseteq{ }^{\omega} \mathbb{R}$, the game $G_{\mathcal{A}}$ is determined, where in the game now, players play a complete real number at each round instead of a single integer. The following conjecture emerged.

Conjecture (The " $\mathbf{A D}_{\mathbb{R}}$ hypothesis") The consistency strength of $\mathrm{AD}_{\mathbb{R}}$ is that of a cardinal $\mu$ that is simultaneously a limit of infinitely many Woodins $\lambda_{n}<\lambda_{n+1} \cdots<$ $\mu$ and of $\mu$-strong cardinals $\kappa_{n}<\lambda_{n}<\kappa_{n+1}$.

The hypothesis is also interesting since (given a mild strengthening of AD), the $\mathrm{AD}_{\mathbb{R}}$-hypothesis is equivalent to the assertion that every set of reals is Suslin. The following is another connection between the worlds of large cardinals and determinacy of games.

$$
\begin{aligned}
& \text { Theorem } 4.8 \text { (Woodin }(\Leftarrow) \text {, Neeman-Steel }(\Rightarrow)) \quad \text { We have } \\
& \qquad \operatorname{Con}\left(\mathrm{ZF}+\mathrm{AD}_{\mathbb{R}}\right) \Leftrightarrow \operatorname{Con}\left(\mathrm{ZFC}+\mathrm{AD}_{\mathbb{R}} \text {-hypothesis }\right) .
\end{aligned}
$$

We continue our cataloging of some more large cardinals through elementary embeddings.

\section{Definition 4.9}

(i) A cardinal $\kappa$ is $\alpha$-supercompact if there is a $j: V \longrightarrow_{e} M$ with ${ }^{\alpha} M \subseteq M$.

(ii) $\kappa$ is supercompact if it is $\alpha$-supercompact for all $\alpha$.

An embedding arising from a measurable cardinal $\kappa$ in general only implies the closure of $M$ under $\kappa$ sequences, so this definition only starts to have bite once $\alpha>\kappa$. The closure under all $\alpha$-sequences is again a considerable strengthening over the "strong" hierarchy of principles. It masks also a telling difference in the kind of embeddings: up to this point the embeddings $j$ have always been "continuous at $\kappa^{+}$" meaning that $\sup \left(j{ }^{\prime \prime} \kappa^{+}\right)=j\left(\kappa^{+}\right)$. If $j$ is a $2^{\kappa}$-supercompact embedding, this fails with $\sup \left(j{ }^{\prime \prime} \kappa^{+}\right)<j\left(\kappa^{+}\right)$occurring. While seeming a rather technical difference this in fact introduces a wide variety of new phenomena. Current inner model theory has a target of producing a good fine structural inner model of a supercompact cardinal, but this target is not yet met. It can do so for strong cardinals, Woodin cardinals, Woodin limit of Woodin cardinals, but although we know what the inner models should look like, for example, for measurably Woodin cardinals, we are unable to prove they exist, the chief difficulty being the inability to prove that sufficiently many ultrapowers of the models are well founded in order for their construction to get off the ground. This has been dubbed the "iterability problem."

Woodin has recently proposed versions of inner models constructed from a single supercompact cardinal (dubbed "Ultimate $L$ ") that would allow the importation to the model of witnesses for all large cardinals, including even larger ones, that may exist in $V$, in one stroke. However, establishing these models with the relevant properties is still a work in progress.

Continuing onward we come to such larger cardinals, the extendible cardinals of Solovay, Reinhardt, and Kanamori [34].

\section{Definition 4.10}

(i) A cardinal $\kappa$ is $\alpha$-extendible if there are $\lambda, j$ with $j: V_{\kappa+\alpha} \longrightarrow_{e} V_{\lambda+\alpha} \wedge$ $\operatorname{cp}(j)=\kappa$.

(ii) $\kappa$ is extendible if it is $\alpha$-extendible for all $\alpha$. 
Even 1-extendibility is a strong concept.

Lemma 4.11 If $\kappa$ is 1-extendible, then it is superstrong (and there are many such below it).

Lemma 4.12 If $\kappa$ is extendible, it is supercompact; $\kappa$-extendible implies $V_{\kappa} \prec \Sigma_{3} V$.

Ascending further we find the following.

Definition 4.13 A cardinal $\kappa$ is huge if there is $j: V \longrightarrow_{e} M$ with $\operatorname{cp}(j)=$ $\kappa \wedge{ }^{j(\kappa)} M \subseteq M$.

However if we try to maximize the extendibility properties we run into inconsistency. Let $\mathscr{L}_{\dot{\epsilon}, j}$ be the usual language with an additional class predicate $j$; let $\mathrm{ZFC}_{j}$ be a formulation of the axioms with the additional predicate symbol allowed into all instances of separation and collection.

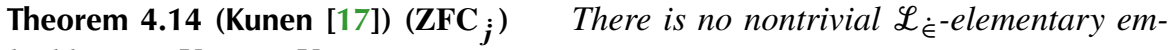
bedding $j: V \longrightarrow_{e} V$.

It is unknown whether AC is necessary for this theorem (see below). The proof of Kunen's theorem is actually a direct ZFC result about sets.

Theorem 4.15 (Kunen) (ZFC) There is no nontrivial elementary embedding $j: V_{\lambda+2} \longrightarrow_{e} V_{\lambda+2}$.

The 2 is an essential artefact of the argument. That there may be a nontrivial $j: V_{\lambda+1} \longrightarrow_{e} V_{\lambda+1}$ is not known to be inconsistent; if this is to be the case, then $\kappa_{0}=\operatorname{cp}(j)<\lambda$, and it can be shown that $\lambda$ has cofinality $\omega$, being $\sup \left\{\kappa_{0}, j\left(\kappa_{0}\right), j j\left(k_{0}\right), \ldots\right\}$. There are now several proofs of Kunen's theorem (see [12]).

For such $V_{\lambda+1}$ the model $L\left(V_{\lambda+1}\right)$ and its possible elementary embeddings has become an object of study and is likely to be significant. Likewise, recent work of Woodin has focused attention on the possibility of an embedding of $V$ to itself being consistent with ZF without choice. Generalizations of such principles are thus beyond the consistency strength of large cardinals considered with AC. It is perhaps then the case, that there is a choiceless realm of strong axioms or large cardinals, lying in a region where $\mathrm{AC}$ fails, thus between the AC-world and inconsistency. It is an interesting observation that there are no deep proofs of the inconsistency of any putative large cardinal axiom yet proposed: usually a half a page at most is needed to see off any inconsistent candidate. So perhaps instead of this picture of all inconsistency proofs being of a simple nature, the $\left(\mathrm{ZF}_{j}\right)$-version of Kunen's theorem, if true, may be the first example where hard work has to be done.

\section{References}

[1] Banach, S., "Uber additive massfunktionen in alstrakten Mengen," Fundamenta Mathematicae, vol. 15 (1930), pp. 97-101. 215

[2] Davis, M., "Infinite games of perfect information," pp. 85-101 in Advances in Game Theory, vol. 52 of Annals of Mathematics Studies, Princeton University Press, Princeton, 1964. Zbl 0133.13104. MR 0170727. 216, 231 
[3] Devlin, K. J., Constructibility, vol. 6 of Perspectives in Mathematical Logic, Springer, Berlin, 1984. MR 0750828. DOI 10.1007/978-3-662-21723-8. 226

[4] Devlin, K. J., and R. B. Jensen, "Marginalia to a theorem of Silver," pp. 115-42 in $\models$ ISILC Logic Conference (Kiel, 1974), edited by A. Oberschelp, G. H. Müller, and K. Potthoff, vol. 499 of Lecture Notes in Mathematics, Springer, Berlin, 1975. MR 0480036. 221

[5] Dodd, A. J., and R. B. Jensen, "The core model," Annals of Mathematical Logic, vol. 20 (1981), pp. 43-75. Zbl 0457.03051. MR 0611394. DOI 10.1016/0003-4843(81)90011-5. 225

[6] Friedman, H. M., "Higher set theory and mathematical practice," Annals of Mathematical Logic, vol. 2 (1970), pp. 325-27. Zbl 0215.32702. MR 0284327. 216, 231

[7] Gödel, K., The Consistency of the Continuum Hypothesis, vol. 3 of Annals of Mathematics Studies, Princeton Univ. Press, Princeton, 1940. MR 0002514. 220

[8] Harrington, L., "Analytic determinacy and 0"," Journal of Symbolic Logic, vol. 43 (1978), pp. 685-93. MR 0518675. DOI 10.2307/2273508. 221

[9] Hanf, W., "Incompactness in languages with infinitely long expressions," Fundamenta Mathematicae, vol. 53 (1964), 325-334. 215

[10] Jech, T., Set Theory, 3rd millennium edition, revised and expanded, Springer Monographs in Mathematics, Springer, Berlin, 2003. MR 1940513. 219, 221

[11] Jensen, R. B., "Inner models and large cardinals," Bulletin of Symbolic Logic, vol. 1 (1995), pp. 393-407. Zbl 0843.03029. MR 1369169. DOI 10.2307/421129. 214, 220

[12] Kanamori, A., The Higher Infinite: Large Cardinals in Set Theory from Their Beginnings, 2nd edition, Springer Monographs in Mathematics, Springer, New York, 2003. MR 1994835. 219, 222, 237, 239

[13] Kechris, A. S., "Homogenous trees and projective scales," pp. 33-73 in Cabal Seminar 77-79, edited by D. A. Martin, A. Kechris, and Y. Moschovakis, vol. 839 of Lecture Notes in Mathematics, Springer, Berlin, 1981. MR 0611165. 233, 234

[14] Koellner, P., "Large cardinals and determinacy," in Stanford Encyclopedia of Philosophy, 2013, http://plato.stanford.edu/entries/large-cardinals-determinacy. 218

[15] Koellner, P., and W. H. Woodin, "Large cardinals from determinacy," pp. 1951-2120 in Handbook of Set Theory, Vols. 1-3, edited by M. Foreman, A. Kanamori, and M. Magidor, Springer, Dordrecht, 2010. MR 2768702. DOI 10.1007/978-1-4020-5764-9_24. 219

[16] Kunen, K., "Some applications of iterated ultrapowers in set theory," Annals of Mathematical Logic, vol. 1 (1970), pp. 179-227. Zbl 0236.02053. MR 0277346. 223

[17] Kunen, K., "Elementary embeddings and infinitary combinatorics," Journal of Symbolic Logic, vol. 36 (1971), pp. 407-13. MR 0311478. 239

[18] Magidor, M., "Representing simple sets of ordinals as countable unions of sets in the core model," Transactions of the American Mathematical Society, vol. 317 (1990), pp. 91-126. MR 0939805. DOI 10.2307/2001455. 226

[19] Martin, D. A., "Measurable cardinals and analytic games," Fundamenta Mathematicae, vol. 66 (1969), pp. 287-91. Zbl 0216.01401. MR 0258637. 217, 221

[20] Martin, D. A., "Borel determinacy," Annals of Mathematics (2), vol. 102 (1975), 363-71. Zbl 0336.02049. MR 0403976. 231

[21] Martin, D. A., "Infinite games," pp. 363-71 in Lehto and Olli, editors, Proceedings of the International Congress of Mathematicians, vol. 1, Academia Scientiarum Fennica, 1980. 217

[22] Martin, D. A., and R. M. Solovay, "A basis theorem for $\Sigma_{3}^{1}$ sets of reals," Annals of Mathematics (2), vol. 89 (1969), pp 138-59. Zbl 0176.27603. MR 0255391. 233

[23] Martin, D. A., and J. R. Steel, "A proof of projective determinacy," Journal of the American Mathematical Society, vol. 2 (1989), pp. 71-125. Zbl 0668.03021. MR 0955605. DOI 10.2307/1990913. 218, 234 
Large Cardinals, Inner Models, and Determinacy: An Introductory Overview 241

[24] Mitchell, W. J., "Ramsey cardinals and constructibility," Journal of Symbolic Logic, vol. 44 (1979), pp. 260-66. Zbl 0409.03033. MR 0534574. DOI 10.2307/2273732. 227

[25] Mitchell, W. J., "Jónsson cardinals, Erdôs cardinals, and the core model," Journal of Symbolic Logic, vol. 64 (1999), pp. 1065-86. MR 1779751. DOI 10.2307/2586619. 227

[26] Moschovakis, Y. N., Descriptive Set Theory, vol. 100 of Studies in Logic and the Foundations of Mathematics, North-Holland, Amsterdam, 1980. MR 0561709. 216, 227, 228

[27] Mycielski, J., and S. Swierczkowski, "On the Lebesgue measurability and the axiom of determinateness," Fundamenta Mathematicae, vol. 54 (1964), 67-71. 216

[28] Sami, R. L., "Analytic determinacy and $0^{\#}$ : A forcing-free proof of Harrington's theorem," Fundamenta Mathematicae, vol. 160 (1999), pp. 153-59. MR 1706478. 221

[29] Schimmerling, E., and M. Zeman, "Square in core models," Bulletin of Symbolic Logic, vol. 7 (2001), pp. 305-14. Zbl 0992.03062. MR 1860606. DOI 10.2307/2687750. 226, 227

[30] Schimmerling, E., and M. Zeman, "Characterization of $\square_{\kappa}$ in core models," Journal of Mathematical Logic, vol. 4 (2004), pp. 1-72. MR 2081183. DOI 10.1142/S0219061304000310. 226

[31] Scott, D., "Measurable cardinals and constructible sets," Bulletin de l'Académie Polonaise des Sciences, Série des Sciences Mathématiques, Astrononomiques et Physiques, vol. 9 (1961), pp. 521-24. Zbl 0154.00702. MR 0143710. 215, 223

[32] Simpson, S. G., Subsystems of Second Order Arithmetic, Perspectives in Mathematical Logic, Springer, Berlin, 1999. MR 1723993. DOI 10.1007/978-3-642-59971-2. 220

[33] Solovay, R., "Measurable cardinals and the continuum hypothesis," Notices of the American Mathematical Society, vol. 12 (1965), 132. 216

[34] Solovay, R. M., W. N. Reinhardt, and A. Kanamori, "Strong axioms of infinity and elementary embeddings," Annals of Mathematical Logic, vol. 13 (1978), pp. 73-116. Zbl 0376.02055. MR 0482431. DOI 10.1016/0003-4843(78)90031-1. 235, 238

[35] Steel, J. R., The Core Model Iterability Problem, vol. 8 of Lecture Notes in Mathematical Logic, Springer, Berlin, 1996. Zbl 0864.03035. MR 1480175. DOI 10.1007/978-3-662-22485-4. 225

[36] Ulam, S., "Zur Masstheorie in der allgemeinen Mengenlehre," Fundamenta Mathematicae, vol. 16 (1930), 140-150. 215

[37] Welch, P. D., "Some remarks on the maximality of inner models," pp. 516-40 in Logic Colloquium '98 (Prague, 1998), vol. 13 of Lecture Notes in Logic, Association for Symbolic Logic, Urbana, Ill., 2000. Zbl 0943.03039. MR 1743279. 227

[38] Woodin, W. H., "The continuum hypothesis, I," Notices of the American Mathematical Society, vol. 48 (2001), pp. 567-76. MR 1834351. 218

[39] Woodin, W. H., "The continuum hypothesis, II," Notices of the American Mathematical Society, vol. 48 (2001), pp. 681-90. MR 1842471. 218

\section{Acknowledgments}

The author would like to warmly thank the organizers of the Birkbeck meeting for the opportunity to give these lectures. The author's work was supported by the Isaac Newton Institute under a long-term fellowship in the program "Syntax and Semantics." 
School of Mathematics

University of Bristol

Bristol BS8 1TW

England

and

Isaac Newton Institute for Mathematical Sciences

Cambridge CB3 OEH

England

p.welch@bristol.ac.uk

www. maths.bris.ac.uk/ mapdw 